\title{
Multi-wavelength study in the region of IRAS 16571-4029 and 16575-4023 sources
}

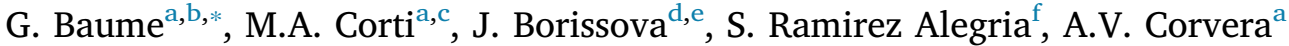 \\ ${ }^{\text {a }}$ Facultad de Ciencias Astronómicas y Geofísicas, UNLP, Argentina \\ ${ }^{\mathrm{b}}$ Instituto de Astrofísica de La Plata CONICET-UNLP, Argentina \\ ' Instituto Argentino de Radioastronomía, CONICET-CICPBA, Argentina \\ d Instituto de Física y Astronomía, Universidad de Valparaíso, Chile \\ ${ }^{\mathrm{e}}$ Millennium Institute of Astrophysics (MAS), Santiago, Chile \\ ${ }^{\mathrm{f}}$ Centro de Astronomía (CITEVA), Universidad de Antofagasta, Chile
}

\section{A R T I C L E I N F O}

\section{Keywords:}

Stars: early type

Stars: pre-main sequence

Galaxy: structure

ISM: HII regions

\begin{abstract}
A B S T R A C T
We studied the stellar populations and their associated interstellar medium structures located in the region of IRAS 16571-4029 and 16575-4023 sources, aiming to evaluate their distance and their main physical parameters. We focused our attention on the embedded cluster DBS 113 and its associated HII region RCW 116B.

For the study, optical images were obtained using wide band and narrow band filters (ugri and OIII, OIII $C \mathrm{H}_{\alpha}$ $H_{\alpha C}$ SII respectively) together with infrared ( $K$ band) spectra of five stars. in the region of DBS 113 . These data were complemented with available bibliographic information and photometric multi-band data from several surveys at optical (VPHAS + ), infrared (2MASS, VVV, WISE) and radio (SGPS, PMN) spectral ranges. Astrometric and photometric data from GAIA DR2 in the region were also taken into account.

Our speectroscopic and photometric study allowed to identify near to 20 early type (OB) stars, about 20 objects with infrared excess, and 10 candidates to young stellar objects in the region of DBS 113. We built their stellar energy distributions ranging from 0.3 to $22 \mu \mathrm{m}$. Our data also suggest an abnormal behavior of the reddening law $\left(R_{V}=4.0\right)$ in the region of this cluster. Besides, we obtained a corresponding spectrophotometric distance from optical/infrared data which was consistent with the distance value computed from GAIA measurements $(d \sim 2 \mathrm{kpc})$. This value locates this cluster at the inner side of the Sagittarius-Carina Galactic arm and it is different from the traditionally adopted kinematic distance of 1-1.2 kpc, based on hydrogen recombination line at millimeter wavelengths. This difference was explained by the presence of a hydrogen expanding shell with a central velocity relative to the molecular cloud. The new proposed distance allowed to estimate more precisely the parameters of the HII region RCW 116B and to describe the energetic balance among the emission of most massive stars, the ionization of the region and the heated dust. Regarding the IRAS 16575-4023 source, we found that the brightest star in this region is a foreground object. We also identified a set of OB-stars candidates with high absorption values $\left(A_{V} \sim 23-26\right)$, located behind the molecular cloud AGAL G345.336 + 01.021.
\end{abstract}

\section{Introduction}

Several small star formation regions in the Milky Way have only been identified or have poor estimated parameters. In particular, their distances are usually based on radial velocity measurements of structures in the interstellar medium (ISM) associated with them. These structures could be bubbles or HII regions (Caswell and Haynes 1987, Lebrón et al. 2001) and the associated measurements often have important uncertainties (Burton 1988; Fich et al. 1989; Russeil et al. 2017) since they are based and strongly depend on Galactic rotation models. Even more, the peculiar streaming motions in the ISM may be an even more important source of uncertainty (Fish et al., 2003). In particular, the kinematic distance estimations are usually less reliable along lines of sight near the Galactic center (Dickey et al. 2003; Clemens 1985). Additionally, in the first and in the fourth quadrant there is ambiguity in the distance value that corresponds to each measured radial velocity. An example is the embedded cluster DBS 179 (Dutra et al., 2003) located in the direction of the H II region G347.6+0.2. A distance of

\footnotetext{
* Corresponding author.

E-mail address: gbaume@fcaglp.unlp.edu.ar (G. Baume).
} 
7.9 kpc was estimated by Borissova et al. (2008) based on JHK photometric measurements and infrared (IR) classification of O type stars. Conversely, using submillimeter observations, Huang et al. (1999) obtained a radial velocity of $V_{L S R}=-95.2 \pm 0.1 \mathrm{~km} \mathrm{~s}^{-1}$ for this region. Using the Fich et al. (1989) rotation model, this value corresponds to the kinematic distances $6.2 \pm 0.1 \mathrm{kpc}$ or $10.4 \pm 0.1 \mathrm{kpc}$ as nearest and farthest solutions, respectively. Later, (Davies et al., 2012) considered a distance of $9 \mathrm{kpc}$ mainly based on HI data. Therefore, it is necessary to look for independent methods to estimate the distances to these regions in order to have a better knowledge of their physical properties. This is important to outline the general structure of our Galaxy. Additionally, the multi-wavelength approach provides a more detailed and precise picture of the stellar populations and especially of their interaction with the surrounding environment. There are several public surveys which can be used for this purpose.

In this work, we focused our attention on IRAS 16571-4029 and 16575-4023 sources. They are two emerging IR sources of the molecular cloud G345.5 + 1.0 and our aim is to evaluate their main parameters to obtain a more precise picture of their physical state. The IRAS 16571-4029 source is associated with the HII region RCW 116B and the embedded cluster DBS 113 (Dutra et al., 2003). This source has several previous studies at different spectral ranges. They include dedicated IR studies of the cluster as those carried out by Verma et al. (2003); Bik et al. (2005, 2006) and Roman-Lopes (2007), and several global works at millimetric radio waves that provided information of the region. On the one hand, most of these works adopted kinematic distances from 1.8 to $2.0 \mathrm{kpc}$ for the clumps associated with the molecular cloud (Bronfman et al. 1996, López et al. 2011, Wienen et al. 2015, Figueira et al. 2019). On the other hand, other works provided values ranging from 1.0 to $1.2 \mathrm{kpc}$ (Caswell and Haynes 1987, Bik et al. 2005; 2006, Roman-Lopes 2007). In particular, (López et al., 2011) obtained an intermediate distance value of $1.8 \mathrm{kpc}$ as the average of several molecular clumps in the region and (Morales et al., 2013) adopted this value for DBS 113. Figueira et al. (2019) drew attention on these discrepancies, which additionally motivated us to re-examine the distance estimations in the region.

To achieve our objectives, we employed a multi-wavelength approach based on our own observations complemented with the information provided by several surveys and the specific texts on this subject. In particular, we used $u$ band data and IR spectroscopic observations to solve degenerated solutions in the analysis.

The paper is organised as follows. In Section 2 we describe the used data sources including our observations and bibliographic ones. Section 3 presents the applied reduction procedures of our observations. Section 4 describes the analysis performed on the data and the main stellar and ISM parameters obtained. In Section 5 we describe the ISM properties in the direction of the IRAS sources. Finally, in

Table 1

Detail of scientific frames acquired using GMOS-S and selected from VSA.

\begin{tabular}{lll}
\hline & \multicolumn{2}{l}{ Exposure times $[\mathrm{s}]$} \\
\cline { 2 - 3 } Filter & Long & Short \\
\hline$u$ & $600(3)$ & $60(3)$ \\
$g$ & $5(3)$ & $1(3)$ \\
$r$ & $5(3)$ & $1(3)$ \\
$i$ & $3(6)$ & $1(3)$ \\
OIII & $100(3)$ & $10(3)$ \\
OIII & $100(3)$ & $10(3)$ \\
$H_{\alpha}$ & $50(3)$ & $5(3)$ \\
$H_{c c}$ & $50(3)$ & $5(3)$ \\
$S I I$ & $100(3)$ & $10(3)$ \\
$J$ & 10 & - \\
$H$ & 10 & - \\
$K$ & 10 & - \\
\hline
\end{tabular}

Note: Number in round brackets indicate the amount of used frames.
Sections 6 and 7, we discuss our results and we draw our conclusions respectively.

\section{Data sources}

\subsection{Optical data}

We used the point sources catalog of the VST Photometric $H_{\alpha}$ Survey of the Southern Galactic Plane and Bulge (VPHAS +; Drew et al. 2014). This catalog includes photometry at the ugriH $H_{\alpha}$ bands. We selected those point sources located in a region of $15^{\prime} \times 15^{\prime}$ centered at the middle point between the IRAS $16571-4029$ and $16575-4023$ sources. This region was identified as our working field of view (WFOV; see Fig. 1)

Direct images were also obtained in April 2018 with the Gemini Multi-Object Spectrograph (GMOS) of Gemini-South (Cerro Pachon, Chile). In this observation run we used wide band filters ugri and narrow band filters $\mathrm{OIII}, \mathrm{OIII}_{G} \mathrm{H}_{\omega}, \mathrm{H}_{\alpha C}$, SII (hereafter identified as NB). See Hook et al. (2004) and Gemini webpage ${ }^{1}$ for instrument details. The GMOS images covered a field of view (FOV) of approximately $5.5^{\prime} \times 5.5^{\prime}$ and were centered in the cluster DBS 113 (see Fig. 3). Three or six dithered exposures were taken per filter (see Table 1) and were shifted 12" to fill in the gaps between the detectors and to remove cosmic rays or bad pixels. Our images were taken under photometric conditions with seeing values ranging from 0.65 " to 0.85 ". We used a $2 \times 2$ binning, giving a detector scale of 0.16 "/pixel (Program GS2018A-Q-323, PI: Baume).

\subsection{Infrared data}

We used the point sources catalog of the Two Micron All Sky Survey (2MASS; Skrutskie et al. 2006), which provides photometric information at the JHK bands to select point sources located in the WFOV. We also obtained the JHK stacked images of the Vista Variables in the Vía Láctea (VVV; Minniti et al. 2010, Saito et al. 2012) survey. These images were downloaded from the VISTA Science Archive (VSA web$s^{2} \mathrm{e}^{2}$ ). The selected images were obtained in 26th March, 2010 and they also covered the previously indicated WFOV (see Fig. 1).

Spectroscopic images of five bright objects located in the region of DBS 113 were also obtained in 13th June, 2015 as part of the 095.D0694(A) ESO program. These objects are identified as blue symbols in Fig. 3 and along the photometric diagrams. For their observation, we used the SOFI@NTT (ESO-La Silla), with a spectral resolution of $R_{\lambda}=$ 1320 in the $K$ band. The resulting signal to noise $(\mathrm{S} / \mathrm{N})$ of the obtained spectra was in the range $\sim 30-95$ and their individual values are indicated in Table 3.

\subsection{Additional data}

We cross-correlated the previous photometric data with the information provided by the AllWISE data Release (Cutri and et al. 2013) and the Gaia Data Release 2 (DR2; Gaia Collaboration et al. 2016; Brown et al. 2018). The AllWISE catalog provides information at 3.6, 4.5, 5.8, and $8.0 \mu \mathrm{m}$ bands, and the GAIA DR2 contains astrometric information together with photometric data at particular bands $G, G_{B P}$ and $G_{R P}$. We also used color images from the Herschel Science Archive ${ }^{3}$ obtained through ALADIN (Bonnarel et al., 2000). These color images were generated as a combination of different images obtained with the Photodetector Array Camera and Spectrometer (PACS) on board of Herschel Space Observatory at 70 and $160 \mu \mathrm{m}$ bands.

\footnotetext{
${ }^{1}$ http://www.gemini.edu/

${ }^{2}$ http://horus.roe.ac.uk/vsa/

${ }^{3}$ http://archives.esac.esa.int/hsa/whsa/
} 


\subsection{Radio data}

We used the information provided by the Southern Galactic Plane Survey (SGPS; McClure-Griffiths et al. 2005) and by the Sydney University Molonglo Sky Survey (SUMSS; Sadler and Hunstead 2001). The former is a survey of $21-\mathrm{cm} \mathrm{H}$ I spectral line emission and continuum. We used the position - velocity datacubes at H I $21-\mathrm{cm}$ line, obtained from the combination data of the Australia Telescope Compact Array (ATCA) and the Parkes single dish telescopes. The angular resolution of these data is $2.2^{\prime}$, has a channel separation of $\Delta v=0.82 \mathrm{~km} \mathrm{~s}^{-1}$ and their final rms noise of a single profile is $\sim 1.6 \mathrm{~K}$ on the brightness temperature $\left(T_{b}\right)$ scale. SUMSS contains radio continuum data at $843 \mathrm{MHz}$ obtained using the Molonglo Observatory Synthesis Telescope (MOST) with a synthesized beam of $43^{\prime \prime} \times 50^{\prime \prime}$ and about $1 \mathrm{mJy}^{\text {beam }}{ }^{-1}$ rms noise.

\section{Reduction procedures}

\subsection{Photometry}

Optical images obtained with GMOS were processed using GEMINIGMOS routines within PYRAF and following the corresponding GMOS Reduction Cookbook. ${ }^{4}$ We applied the appropriate bias and flat-field corrections using bias and flat-field images acquired from the Gemini Science Archive (GSA) and we stacked the exposures obtained with the same filter and exposure time.

As a next step, we performed the photometric measurement over the optical and IR images following a similar procedure used in Corti et al. (2016). Briefly, instrumental magnitudes were obtained using the point spread function (PSF) method (Stetson 1987) and the corresponding photometric tables were aperture-corrected for each filter to carry them to a final aperture size of 17 pixels in radius. All resulting tables were combined using DAOMASTER code (Stetson 1992), obtaining one set for $u_{g r i H}+$ NB bands and other for JHK bands. For ugri bands, the calibration in flux was done using the information of 91 common stars in the VPHAS + catalog. For the remaining NB filters, the corresponding zero point values were estimated in relation to the $H_{\alpha}$ zero point $\left(h a_{1}\right)$ comparing the respective filters response. In the case of the JHK bands the calibration was carried out using the 2MASS catalog. In all this procedure we used the following transformation equations:

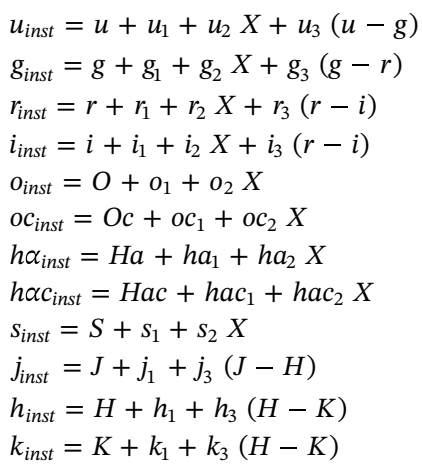

where $u_{g r i H_{\alpha}} J H K$ and $\left(u g r i h_{\alpha} j h k\right)_{i n s t}$ are the VPHAS + and 2MASS standard and instrumental magnitudes, respectively, and $X$ is the airmass of the observation. The extinction coefficients $\left(u_{2}, g_{2}, r_{2}, i_{2}\right)$ are given at the Gemini webpage. The other coefficients were computed using the FITPARAMs task of IRAF PHOTCAL package. The obtained values are shown in Table 2.

We compared our near IR photometry with that given by RomanLopes (2007), deriving the luminosity functions (LFs, see Fig. 2) for each band (JHK) using the DBS 113 cluster region data (see Fig. 1), and
Table 2

Calibration coefficients used for optical and infrared observations together with the corresponding rms fit values.

\begin{tabular}{|c|c|c|c|c|c|}
\hline Coeff. & $x=1$ & $x=2$ & $x=3$ & & $r m s$ \\
\hline \multicolumn{6}{|c|}{ VPHAS + calibration } \\
\hline$u_{x}$ & $+1.96 \pm 0.06$ & 0.38 & -0.14 & \pm 0.06 & 0.15 \\
\hline$g_{x}$ & $-2.88 \pm 0.04$ & 0.18 & -0.14 & \pm 0.02 & 0.07 \\
\hline$r_{x}$ & $-2.75 \pm 0.05$ & 0.10 & +0.01 & \pm 0.06 & 0.09 \\
\hline$i_{x}$ & $-2.44 \pm 0.04$ & 0.08 & -0.15 & \pm 0.04 & 0.07 \\
\hline$h a_{x}$ & $+0.64 \pm 0.01$ & $r_{2}$ & - & & 0.06 \\
\hline \multicolumn{6}{|c|}{ Estimations using transmission curves } \\
\hline$o_{x}$ & $h a_{1}-0.306$ & $g_{2}$ & - & & - \\
\hline$o c_{x}$ & $h a_{1}-1.082$ & $g_{2}$ & - & & - \\
\hline$h a c_{x}$ & $h a_{1}-0.089$ & $r_{2}$ & - & & - \\
\hline$s_{x}$ & $h a_{1}+0.401$ & $r_{2}$ & - & & - \\
\hline \multicolumn{6}{|c|}{ 2MASS calibration } \\
\hline$j_{x}$ & \pm 0.02 & - & -0.03 & 0.01 & 0.05 \\
\hline$h_{x}$ & \pm 0.01 & - & +0.06 & 0.01 & 0.06 \\
\hline$k_{x}$ & -2.19 & - & +0.12 & \pm 0.01 & 0.12 \\
\hline
\end{tabular}

excluding objects with high error values $(>0.5)$. The RomanLopes (2007) data revealed a completeness $\sim 40-50 \%$ when their LFs were compared with our ones. On the contrary, our near IR data are about 3 magnitudes deeper (at each band) than Roman-Lopes (2007) ones. We also see in Fig. 2 that the number of stars per bin begins to decrease at $J \sim 19, H \sim 17$ and $K \sim 15.5$. Therefore, we considered our data are complete up to these values.

\subsection{Astrometry}

We used a similar procedure as in Baume et al. (2009). That is, since the information provided by the World Coordinate System (WCS) was only available for VVV images, we used the ALADIN tool together with the VPHAS + and 2MASS catalogs to obtain a reliable astrometric calibration for our optical images. The resulting root mean square (rms) of the residuals in the positions were $\sim 0.10^{\prime}-0.15$ ', which is about the astrometric precision of the catalogs employed.

\subsection{Spectroscopy}

We followed the standard procedures to reduce the spectroscopic data, for more details see Moorwood et al. (1998), Chené et al. (2012) and Chené et al. (2013). In short, we subtracted nodding observations from one another to remove the bias level and sky emission lines. Then flat fielding, spectrum extraction, wavelength calibration and correction for atmospheric absorption of all spectra were done using IRAF tasks. Finally, all spectra were rectified using a low-order polynomial fit to a wavelength interval that was assumed to be pure continuum. In particular, object ID 127 was observed twice using different slit positions and both observations were used to verify the reliability of the reduction and the wavelength calibration processes.

\subsection{Final catalogs}

We used the sTiLTs ${ }^{5}$ tool to manipulate tables and to crosscorrelate the optical and IR data. We then obtained a catalog with astrometric and photometric information (ugri $+\mathrm{NB}+J H K+W_{1} W_{2} W_{3} W_{4}+$ $G G_{B P} G_{R P}$ bands) for about 44,600 objects in the WFOV. The photometric errors in the catalog were those provided by the DAOРHOT, DAOMASTER codes and the corresponding source catalogs. The full catalog (Table 3) is only available on-line at the CDS. 
Table 3

Selected objects in the region of IRAS 16571-4029 source.

\begin{tabular}{|c|c|c|c|c|c|c|c|c|}
\hline$I D$ & VPHAS + & 2MASS & RL07 & $\alpha_{J 2000}$ & $\delta_{J 2000}$ & $K$ & $\mathrm{SpT}^{1}$ & $\mathrm{~S} / \mathrm{N}$ \\
\hline 40 & 1112a-11-05461112b-21-1037 & $17003247-4033125$ & 9 & $17: 00: 32.5$ & $-40: 33: 12.5$ & 10.90 & B4-5 & 29 \\
\hline 69 & 1112a-11-04871112b-21-0971 & $17003449-4033403$ & 3 & $17: 00: 34.5$ & $-40: 33: 40.2$ & 9.29 & 08-9 & 95 \\
\hline 127 & 1112a-11-15171112b-21-0976 & $17003386-4033382$ & 2 & $17: 00: 33.9$ & $-40: 33: 38.1$ & 9.04 & O9-B1 & $32,40^{2}$ \\
\hline 2395 & 1112a-11-04481112b-21-0916 & - & 23 & 17:00:33.5 & $-40: 34: 03.1$ & 11.84 & middle B & 35 \\
\hline 9116 & - & - & - & $17: 00: 33.6$ & $-40: 33: 46.0$ & 12.53 & middle B & 37 \\
\hline
\end{tabular}

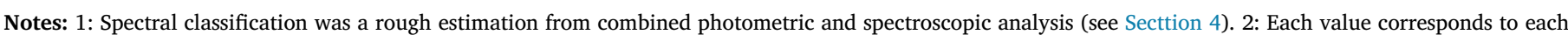

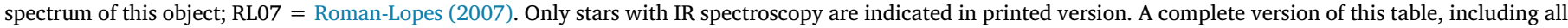
the studied photometric bands and the DBS 113 members photometrically selected, is available on-line at the CDS.

\section{Stellar populations at IRAS sources}

To study the behavior of the stellar populations in the zone of the IRAS $16571-4029$ and 16575-4023 sources, we selected particular circular regions. These regions are indicated in Fig. 1. In the case of IRAS 16571-4029 source the stellar counterpart was clear; however, in the case of IRAS 16575-4023 source it was hard to identify. Therefore, for this latter source we considered two regions (identified as A and B; see Fig. 4). The former was chosen based on the location of the brightest star in the area, while the latter was chosen considering the region where the molecular cloud AGAL G345.336+01.021 (Wienen et al., 2015) was identified. The adopted centers and radius values for each circle are presented in Table 4. Then, using our catalog, we built the color-magnitude and two-color diagrams (CMDs and TCDs, respectively) for each region. They are presented in Figs. 7, 9 and 11.

All the photometric diagrams clearly revealed the presence of differential reddening and since we were dealing with a high absorption region, the optical ones presented a much lower amount of objects than the IR ones. On one hand, optical photometric diagrams allowed us to obtain a better distance estimation using the traditional main sequence (MS) fitting method. Nevertheless, it must be noticed that, for very faint objects at optical bands with large error values, these photometric diagrams can be used only to get a general picture of the cluster parameters as the foreground color excesses and/or distance, but not to follow very precise behaviors of particular objects. On the other hand, the upper MS has an almost vertical shape over the IR CMDs not allowing to estimate precise distance values. However, these diagrams provided an estimation of the foreground color excess of young stellar populations.

Table 4

Parameters of studied IRAS sources.

\begin{tabular}{|c|c|c|}
\hline \multirow{3}{*}{$\begin{array}{l}\text { IRAS } \\
\text { ATLASGAL } \\
\text { Optic }\end{array}$} & $16571-4029$ & \multirow{3}{*}{$\begin{array}{l}16575-4023(\mathrm{~B}) \\
345.336+01.021\end{array}$} \\
\hline & $345.214+01.025$ & \\
\hline & DBS $113 \equiv$ RCW $116 \mathrm{~B}$ & \\
\hline$\alpha_{J 2000}$ & $17: 00: 35.0$ & $17: 01: 02.0$ \\
\hline$\delta_{J 2000}$ & $-40: 33: 44.0$ & $-40: 27: 32.7$ \\
\hline Radius ['] & 1.8 & 0.5 \\
\hline$R_{V}$ & 4.0 & $3.1-4.0$ \\
\hline$E_{B}-V \min$ & 1.8 & $8.3-5.8$ \\
\hline$E_{B-V \max }$ & 4.5 & $14.0-10.0$ \\
\hline$A_{V}$ & $\sim 7$ & $\sim 26-23$ \\
\hline$V_{0}-M_{V}$ & $11.5 \pm 0.2$ & 11.5 \\
\hline$d_{\text {phot }}[\mathrm{kpc}]$ & $2.0 \pm 0.2$ & 2 \\
\hline$\langle P l x\rangle[\mathrm{mas}]$ & $0.49 \pm 0.07(5)$ & - \\
\hline$d_{\text {ast }}[\mathrm{kpc}]$ & $2.0 \pm 0.2(5)$ & - \\
\hline$\left\langle\mu_{\alpha} \cos (\delta)\right\rangle[\mathrm{mas} / \mathrm{yr}]$ & $-0.52 \pm 0.09(7)$ & - \\
\hline$\left\langle\mu_{\delta}\right\rangle[\mathrm{mas} / \mathrm{yr}]$ & $-1.65 \pm 0.07(7)$ & - \\
\hline$V_{R_{L S R}}[\mathrm{~km} / \mathrm{s}]^{a}$ & -15.24 & -15.17 \\
\hline$d_{k i n}[\mathrm{kpc}]^{a}$ & $1.9 \pm 0.7$ & $1.9 \pm 0.7$ \\
\hline$N_{\text {Lystars }}\left[\right.$ photons s $\left.{ }^{-1}\right]$ & $\sim 1.210^{49}$ & - \\
\hline $\mathrm{U}\left[\mathrm{pc} \mathrm{cm}^{-2}\right]^{(b)}$ & $60-68$ & - \\
\hline
\end{tabular}

Notes: Numbers in parentheses indicate the amount of objects considered to compute the corresponding average values. (a): ATLASGAL (Wienen et al., 2015). (b) $U$ values obtained for $5000 \mathrm{~K}$ and $7600 \mathrm{~K}$ in $T_{e}$.

\subsection{IRAS 16571-4029 region}

The obtained spectra of the five stars observed in this region (see Section 2.2) are presented in Fig. 5 together with a nebular spectrum. We noticed that most of the objects and the nebular spectrum present only the $\mathrm{Br} \gamma$ line in emission, suggesting that stellar spectra could be contaminated by nebular emission. Notwithstanding this problem, we were able to carry out a rough spectral classification from combined spectroscopic and photometric data and following an iterative process testing different color excess and distance values.

In this iterative process we mainly considered, on one hand, the comparison of our SOFI spectra with similar resolution $K$-band libraries. Specifically, we used the libraries given by Hanson et al. (1996, 2005) and the "ESO stellar spectral library". This spectral classification was based on the identification and comparison of the He I 2.059 and $2.113 \mu \mathrm{m}$ and $\mathrm{Br}_{\gamma} 2.166 \mu \mathrm{m}$ lines. On the other hand, we checked the location of each star in the $J$ vs. $J-H$ diagram (see Fig. 9b). It was a good indicator of the spectral type in that $J$ should be little affected by possible near IR excess emission. The obtained spectral classification is presented in Table 3. We notice that we were dealing with early type stars as expected for young star members of an embedded cluster.

Three of the stars with spectroscopic observations (IDs 40, 69 and 127) were identified and measured at all optical and near IR bands (ugriJHK; see Table 3). This information allowed to estimate the reddening behavior in this direction, applying the color difference method. We estimated the selective absorption coefficient $\left(R_{V}=A_{V} / E_{B-V}\right)$ computing the color excess ratios at the indicated bands relative to $E_{g_{-} r}$, $E_{g-i}$ and $E_{u-g}$. The obtained results were compared with the corresponding values given by the reddening model of Cardelli et al. (1989) for a B5 V-type star (see Molina Lera et al. 2018). The used stars yield in general large $R_{V}$ values with a dependence on the adopted reference color excess. These facts were interpreted as a consequence of emission features in their spectra (see Fig. 5) and/or uncertainties in $u$ band magnitudes. Therefore, we focused our attention on the behavior of ID 40 object (B4-5 type). This object seems to present no abnormal spectral features and lies outside the region where the nebular emission is most important. Additionally, it was the brightest of the five stars with spectra. These facts would ensure high reliability of the estimated colors and spectral classification for this star.

The results for the three stars are presented in Fig. 6, where $R_{V}$ error values were estimated based on the different results obtained by varying both the spectral classification of each star (in one spectral type) and the reference color excess used in the method. Finally, we adopted a value of $R_{V}=4.0$ as representative for the DBS 113 cluster region and we could compute spectrophotometric color excesses and distances for the five stars with spectroscopic observations. In particular, the location of ID 40 in the $u-g$ vs. $g-r$ diagram (see Fig. 7a) is more consistent with the adopted spectral classification and an abnormal reddening behaviour.

\footnotetext{
${ }^{6}$ https://www.eso.org/sci/observing/tools/standards/IR_spectral_library. html
} 
This procedure allowed us to obtain color excesses in $E_{B-V}$ ranging from 1.8 to 4.2 and $V_{0}-M_{V}$ values between 10.5 to 11.9 depending on the star and the used color index. The spread in color excess values reflects the classic evidence of a young and embedded cluster, whereas the different values in distance are interpreted as a consequence of particular features in the spectral distribution of the considered stars.

As a next step, we selected different kinds of objects using only photometric data. We mainly based our analysis on the color excess range covered by stars with spectral information. The adopted extreme values for color excesses are presented in Table 4 as $E_{B-V \text { min }}$ and $E_{B-V \max }$. Values for the other color indices were computed using the corresponding $r_{x}$ values. These extreme values are indicated also in the photometric diagrams by the location of the shifted ZAMS $\left(E_{I C m i n}\right)$ and the point of the reddening vector $\left(E_{I C \max }\right)$. We also used values of the reddening-free photometric parameters defined as:

$$
\begin{aligned}
& Q_{I R}=(J-H)-\left(r_{J}-r_{H}\right) /\left(r_{H}-r_{K}\right)(H-K) \\
& Q_{J H}=(J-D M)-r_{J} R_{V}(J-H)\left(r_{B}-r_{V}\right) /\left(r_{J}-r_{H}\right) \\
& Q_{H K}=(K-D M)-r_{K} R_{V}(H-K)\left(r_{B}-r_{V}\right) /\left(r_{H}-r_{K}\right) \\
& Q_{J K}=(J-D M)-r_{J} R_{V}(J-K)\left(r_{B}-r_{V}\right) /\left(r_{J}-r_{K}\right)
\end{aligned}
$$

where $r_{J}, r_{H}$ and $r_{K}$ are the relative absorptions $\left(r_{x}=A_{x} / A_{V}\right)$ for $J H K$ bands at the adopted $R_{V}$ value, and DM is the considered distance modulus. Specifically, the identification of probable members of the studied regions and their classification in different categories was made with the following criteria:

- Early MS stars with color excesses in the range adopted above. This is, objects following the conditions: $-0.1<Q_{I R}<0.4, Q_{I C}<0.5$ and $E_{I C \min }<E_{I C}<E_{I C \max }$, with IC $=J-H, H-K$ and $J-K$.

- Objects with IR color excess, identified with the same conditions used for early MS but with $Q_{I R}<-0.1$.

- Objects identified as class I and class II YSOs, following the WISE bands color conditions given by Koenig et al. (2012)

The remaining objects were not classified and they were considered mainly as field stars.

Additionally, we employed the photometric diagrams which involve NB filters computing the color indices $O-O_{C}, H_{\alpha}-H_{\alpha C}$ and $S-H_{\alpha}$ (see Fig. 8). Therefore, we applied a Principal Components Analysis (PCA) over the tri-dimensional space of the color indices to identify objects with line emission symptoms in at least one of these indices.

After trying several distance values, we found the most consistent solution along all the photometric diagrams adopting the $V_{0}-M_{V}=11.5$ for DBS 113 cluster (see Table 4).

The photometric and astrometric information based on GAIA DR2 data on the adopted region for IRAS 16571-4029 source (see Fig. 10) allowed to refine our previous photometric member selection criteria. Otherwise, we applied a PCA over the proper motion diagram to eliminate objects placed further than one normalized standard deviations of the mean proper motion value. Regarding the trigonometric distance, the selected objects had large relative error values ( $\sim 40 \%$ ) in parallax, so we followed the Cantat-Gaudin et al. (2018) procedure to estimate their distance and proper motion. Briefly, we added +0.029 mas to all parallax values and then computed weighted averages over the paralaxes and proper motion values $(\langle P l x\rangle$, $\left\langle\mu_{\alpha} \cos (\delta)\right\rangle$ and $\left\langle\mu_{\delta}\right\rangle$ ). In the case of parallaxes we selected those values with relative errors lower than 1 . The obtained results are presented in Table 4. It could be noticed that the resulting relative error in parallax was lower than $20 \%$ allowing us to estimate the corresponding distance as $d_{a s t}=\langle P l x\rangle^{-1}$ (see Bailer-Jones 2015 for details). This computed distance was consistent with the already adopted one using the stellar spectra and the photometric diagrams, which reinforce our previous analysis.

\subsection{IRAS $16575-4023$ regions}

In this case, the information from GAIA DR2 indicates there are seven stars with parallax and proper motion measures in the region A. However, none of them was photometrically selected as a member. In particular, the brightest star in this region (ID 720) has a $P l x=4.8 \pm 0.5$ mas $(\sim 200 \mathrm{pc})$ revealing that it is a foreground star with no physical relation with the IRAS source.Therefore, we focused our attention on region $\mathrm{B}$ and we followed the object selection procedure explained before for IRAS 16571-4029 region. However, since we did not have deep optical photometric data for this case, we used only IR data. We also identified those objects with no $J$ band data and $H-K>2.1$ as probable reddened early type (OB) stars and represented with red triangles in Fig. 11. The MS fit presented on this figure was done adopting $R_{V} 3.1$ and 4.0 as extreme values and the corresponding color excess and visual absorption values indicated in Table 4. In both cases we kept the same distance value considered for DBS 113 cluster.

\subsection{Stellar parameters}

For a given distance, the parameter $Q_{J H}$ was used to estimate the spectral types of the MS member stars, since this parameter was slightly influenced by the presence of emission lines. This procedure allowed us to compute the number of ionizing photons produced by each cluster star using the models given by Sternberg et al. (2003) considering a Luminosity Class V. Therefore, we could estimate the total number of ionizing photons $\left(N_{\text {Lystars }}\right)$ produced by all the cluster and the corresponding "ionization parameter" $(U$ ) given by Wilson et al. (2013) as:

$U\left[p c \mathrm{~cm}^{-2}\right]=3.24110^{-19} \sqrt[3]{\frac{3 N_{L y ~ s t a r s}\left[\mathrm{~s}^{-1}\right]}{4 \pi \alpha_{2}\left(T_{e}\right)\left[\mathrm{cm}^{3} \mathrm{~s}^{-1}\right]}}$

where $\alpha_{2}\left(T_{e}\right)$ is the total recombination coefficient of hydrogen excluding captures to the ground level. This coefficient was computed using the expression indicated by Spitzer (1978) and the electronic temperature $\left(T_{e}\right)$ values considered for the HII region (see Table 5). These estimations were carried out only for DBS 113 and we found that in this case the $U$ value was mainly defined by objects ID 69 and ID 127 (see Sect. 6.1.1). The obtained $U$ values are indicated in Table 4.

Table 5

\begin{tabular}{|c|c|c|}
\hline & $\begin{array}{l}\text { IRAS 16571-4029 } \\
\text { RCW 116B }\end{array}$ & IRAS $16575-4023$ \\
\hline \multicolumn{3}{|c|}{ Parameters from SUMSS (843 MHz) } \\
\hline$(1, \mathrm{~b})\left[^{\circ}\right]$ & $(345.21,+1.02)$ & $(345.33,+1.02)$ \\
\hline$s_{\nu b g}\left[\mathrm{Jy} \mathrm{beam}^{-1}\right]$ & $0.070 \pm 0.010$ & $0.010 \pm 0.001$ \\
\hline$r m s_{s \nu}\left[\mathrm{Jy} \mathrm{beam}^{-1}\right]$ & 0.031 & 0.009 \\
\hline$S_{\nu}[\mathrm{Jy}]$ & $7 \pm 3$ & $0.3 \pm 0.1$ \\
\hline$s_{\text {Lpeak }}\left[\mathrm{Jy} \mathrm{beam}^{-1}\right]$ & 2.72 & 0.17 \\
\hline iso $_{\min }\left[\mathrm{Jy}\right.$ beam $\left.^{-1}\right]$ & 0.30 & 0.05 \\
\hline$\theta_{a}\left[{ }^{\prime}\right] \times \theta_{b}\left[{ }^{\prime}\right]$ & $1.5 \times 1.3$ & $0.85 \times 0.70$ \\
\hline$\theta_{\text {sph }}\left[{ }^{\prime}\right]$ & 1.2 & $\sim 0$ \\
\hline \multicolumn{3}{|c|}{ Physical ISM parameters } \\
\hline$R_{s}[\mathrm{pc}]$ & $0.35 \pm 0.12$ & - \\
\hline$T_{e}[\mathrm{~K}]$ & $5000^{(1)}-7600^{(2)}$ & - \\
\hline$\alpha_{2}\left[10^{-13} \mathrm{~cm}^{3} \mathrm{~s}^{-1}\right]$ & $4.496^{(1)}-3.219^{(2)}$ & - \\
\hline$N_{e}\left[\mathrm{~cm}^{-3}\right]$ & $1253^{(1)}-1347^{(2)}$ & - \\
\hline$E M\left[10^{5} \mathrm{pc} \mathrm{cm}^{-6}\right]$ & $8.4^{(1)}-9.7^{(2)}$ & - \\
\hline$\mu[\mathrm{pc} \mathrm{cm}-2]$ & $41^{(1)}-43^{(2)}$ & - \\
\hline$M_{H I I}\left[M_{\odot}\right]$ & $11.3^{(1)}-12.2^{(2)}$ & - \\
\hline
\end{tabular}

Main parameters of the studied IRAS sources obtained from the $843 \mathrm{MHz}$ data and complementary information from the literature. Errors computed adopting a distance error of $0.2 \mathrm{kpc}$.

Note: $T_{e}$ values are from Caswell and Haynes (1987) (1) and Quireza et al. (2006b) (2). Superscripts at ISM parameter values indicate adopted $T_{e}$ value for each case 


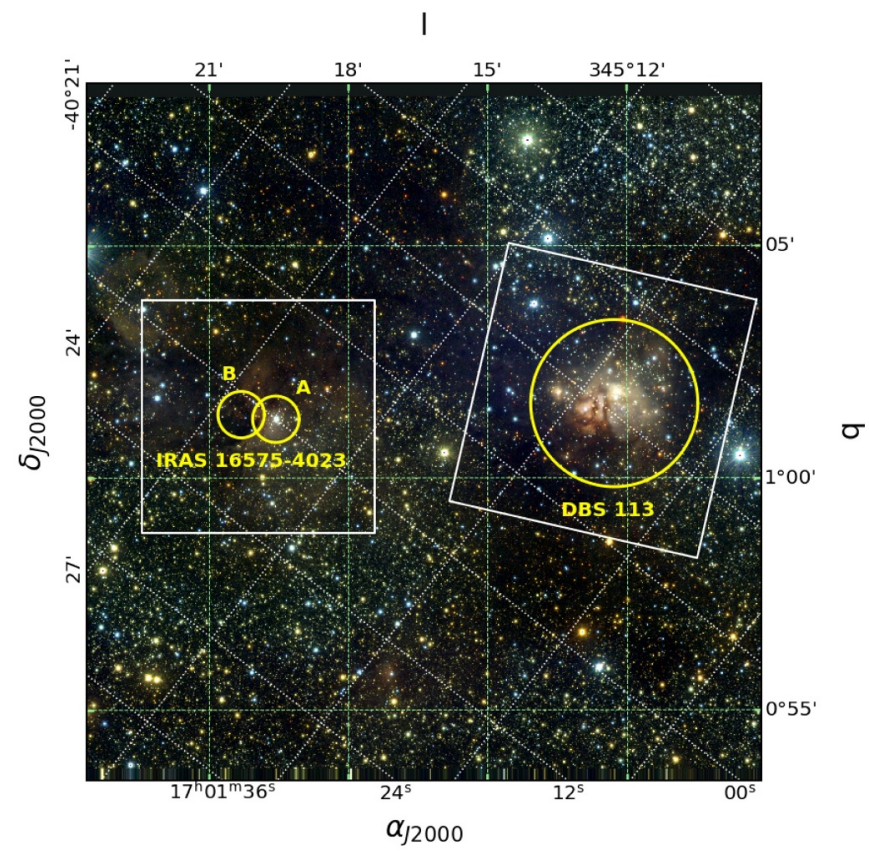

Fig. 1. JHK false-color VVV image of working field of view (WFOV). This field is centered at $\alpha_{J 2000}=17: 00: 49.87, \delta_{J 2000}=-40: 30: 43.7$ and covers $15^{\prime} \times 15^{\prime}$. DBS 113 cluster region and the two selected regions (A and B) associated with the IRAS 16575-4023 source are indicated by yellow circles. These circles indicate the regions used to build the corresponding photometric diagrams of the IRAS sources. White rectangles represent the 5.5' $\times 5.5^{\prime}$ region covered by Gemini observations (see Fig. 3) and the region covered in Fig. 4. (For interpretation of the references to colour in this figure legend, the reader is referred to the web version of this article.)

\section{The ISM in the direction of the IRAS sources}

\subsection{Radio maps}

Radio maps covering approximately the WFOV of the studied region (see Fig. 1) are presented in Figs. 12 and 13. Fig. 12 corresponds to continuum SUMSS observations at $843 \mathrm{MHz}$ and Fig. 13 shows an average of several velocity channels at HI from SGPS. These maps revealed, respectively, clear emission and absorption signatures in the location of IRAS 16571-4029 which are consistent with a source with a continuum temperature higher than the temperature of the ISM placed in front of it. However, in the case of IRAS 16575-4023 only a faint emission centered at region $\mathrm{A}$ is detected at $843 \mathrm{MHz}$, but no evidence of it could be found on any channel of the HI data. Additionally, in Fig. 12 it can be noticed that both IRAS sources show an almost similar shape. This fact was understood as a reflection effect of the instrumental beam and revealing that both sources could be considered with approximately circular shapes. Since the observed profiles are the result of the convolution between the real angular size $\left(\theta_{s p h}\right)$ and the beam angular size $\left(\theta_{B a} \times \theta_{B b}\right)$ of the SUMSS observations, the former could be estimated with the following relation:

$\theta_{s p h}=\sqrt{\left(\theta_{a} \theta_{b}\right)-\left(\theta_{B a} \theta_{B b}\right)}$

where each emission source was considered as a bidimensional gaussian distribution (Fig. 14), and values $\theta_{a}$ and $\theta_{b}$ were the corresponding measured half-power beam width (HPBW) at each principal axis. It must be noticed that this procedure only yielded a reliable result for IRAS 16571-4029 source, since for IRAS $16575-4023$ we obtained a negligible angular value and we considered it as a point source.

The corresponding "flux densities" $\left(S_{\nu}\right)$ for each IRAS source at $843 \mathrm{MHz}$ were obtained using AIPS tools. These values were computed as the difference between the total flux at the source place down to a
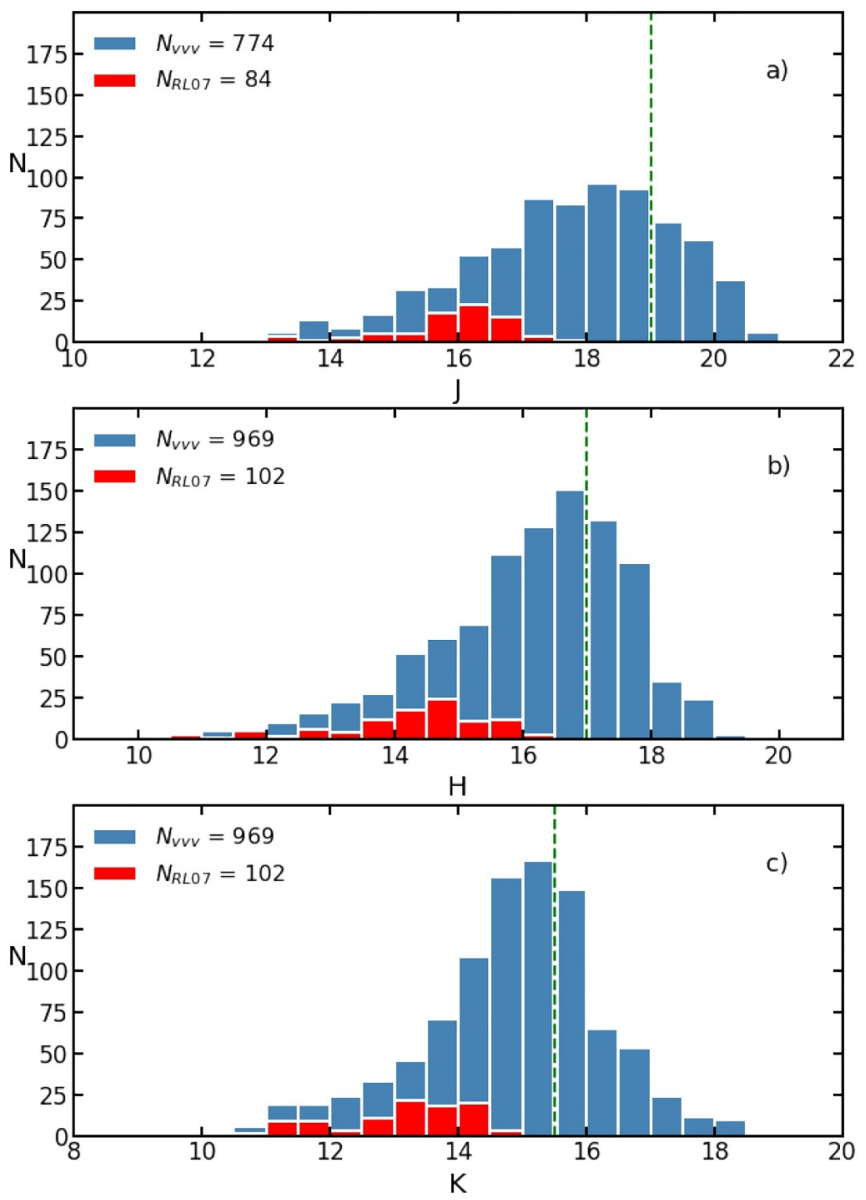

Fig. 2. Luminosity functions for the DBS 113 cluster region derived from our data set (blue) and Roman-Lopes (2007) data (red). Vertical dotted lines indicate adopted limit magnitude values for our photometry. (For interpretation of the references to colour in this figure legend, the reader is referred to the web version of this article.)

minimum value $\left(i s o_{\min }\right)$ and an estimation of its background level $\left(s_{b k g}\right)$. The corresponding background values were estimated averaging the mean flux density values at three different positions near to each source, whereas the rms of the flux density values $\left(r m s_{S_{y}}\right)$ was considered as a measure of the noise. The corresponding parameters for each IRAS source are presented in Table 5.

In Fig. 15 we present a position - velocity map of the SGPS data cube at IRAS 16571-4029 location $\left(l=345.2^{\circ}\right)$. This figure clearly shows the presence of a minimum in brightness extended from $\sim+10$ to $\sim-30 \mathrm{~km} \mathrm{~s}^{-1}$ with a break in the range between $\sim-5$ to $\sim-13 \mathrm{~km} \mathrm{~s}^{-1}$. Conversely, the emission peaks of $\mathrm{H}$ I centered at $\sim-10$ and $\sim-20$ $\mathrm{km} \mathrm{s}^{-1}$ are respectively consistent with the location of the SagittariusCarina and Scutum-Crux arms according to the Vallée (2008) model for the Galactic arms and the Fich et al. (1989) model for the Galactic rotation.

\subsection{Physical ISM parameters}

The $843 \mathrm{MHz}$ data in the region of both IRAS sources combined with information from previous works in this region allowed to estimate several physical ISM parameters. Additionally, according to their far IR (FIR) colors (Bronfman et al., 1996), IRAS 16571-4029 and 16575-4023 sources are considered as two ultracompact H II regions. Therefore, we used the indicated data and the expressions presented by Mezger and Henderson (1967) to compute the "electron density" $\left(N_{e}\right)$, the "mass of ionized hydrogen" $\left(M_{H I I}\right)$ and the "emission measure" $(E M)$. The used 

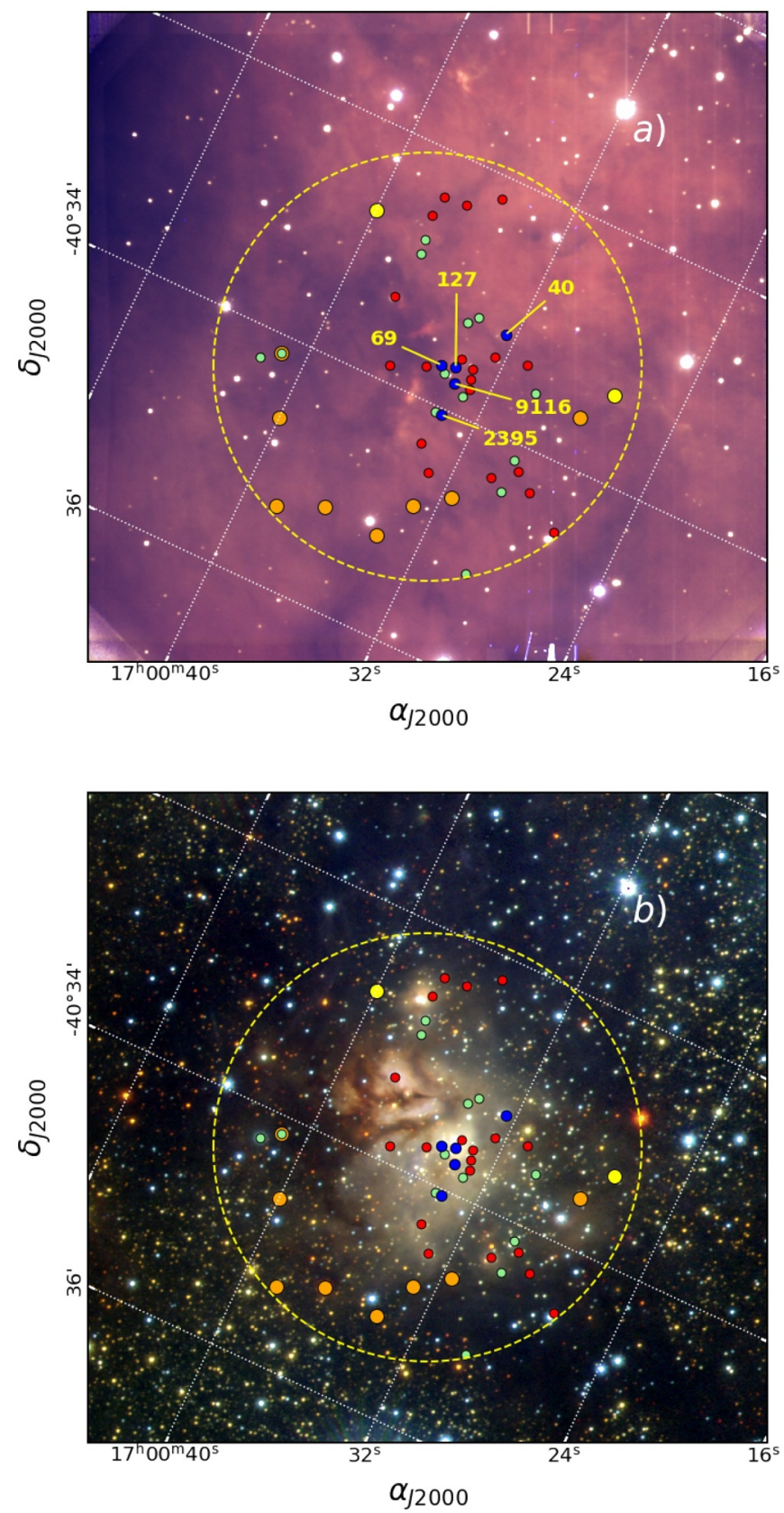

Fig. 3. Color images of the embedded cluster DBS 113 region with the FOV covered with GMOS observations. RGB colors correspond to $u$, OIII, $H_{\alpha}, S I I$ filters (panel a) and to $J H K$ ones (panel b). Blue symbols represent stars with spectroscopic observations (see Section 2.2 and Fig. 5), green symbols correspond to MS stars and red ones to stars with probable IR excess. Yellow and orange circles indicate respectively Class I and II YSOs which were identified using WISE colors. See Section 4.1 for details. Yellow dashed circle indicates the selected region for the photometric analysis. In panel a the identification number for stars with spectroscopic observations is included. (For interpretation of the references to colour in this figure legend, the reader is referred to the web version of this article.)

equations were:

$$
\begin{aligned}
N_{e}\left[\mathrm{~cm}^{-3}\right]= & \mu_{1} a^{0.5} 635.1\left(T_{e}\left[10^{4} \mathrm{~K}\right]\right)^{0.175}(\nu[\mathrm{GHz}])^{0.05} \times \\
& \times\left(S_{\nu}[\mathrm{Jy}]\right)^{0.5}(d[\mathrm{kpc}])^{-0.5}\left(\theta_{G}[\text { arcmin }]\right)^{-1.5} \\
M_{H I I}\left[M_{\odot}\right]= & \mu_{2} a^{0.5} 0.3864\left(T_{e}\left[10^{4} \mathrm{~K}\right]\right)^{0.175}(\nu[\mathrm{GHz}])^{0.05} \times \\
& \times\left(S_{\nu}[\mathrm{Jy}]\right)^{0.5}(d[\mathrm{kpc}])^{2.5}\left(\theta_{G}[\text { arcmin }]\right)^{1.5}
\end{aligned}
$$
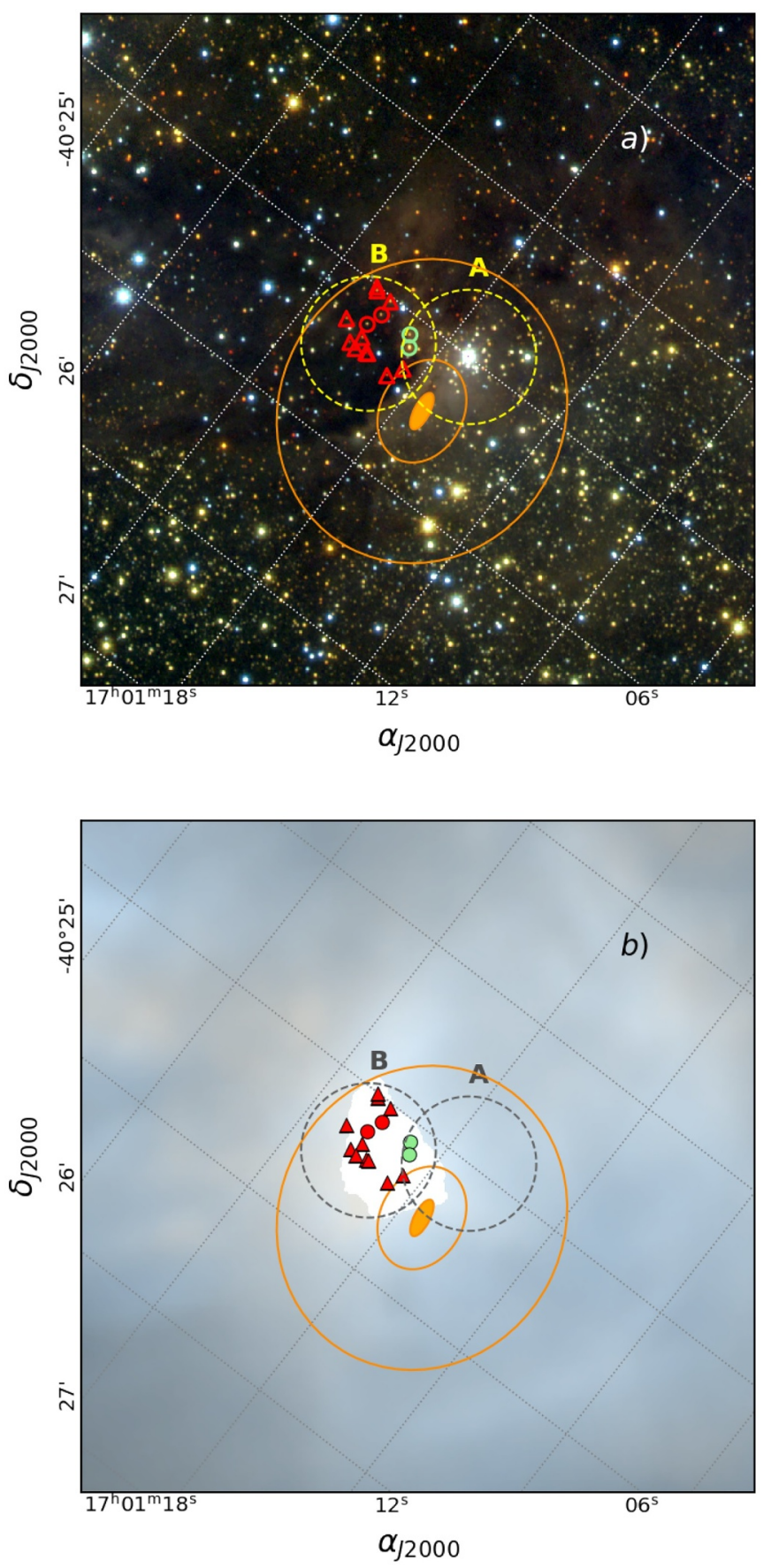

Fig. 4. Color images of the region centered in the IRAS 16575-4023 source using JHK bands (panel a) and PACS/Herschel $70 \mu \mathrm{m}$ and $160 \mu \mathrm{m}$ bands (panel b). Symbols as in Fig. 3 and red triangles represent probable early type stars (see Section 4.2 and Fig. 11). Dashed circles indicate both studied regions. Location and error ellipse of the IRAS source is presented with a filled orange symbol. Larger orange ellipses represent IRAS angular resolutions at $12 \mu \mathrm{m}$ (30") and $100 \mu \mathrm{m}$ (2'). (For interpretation of the references to colour in this figure legend, the reader is referred to the web version of this article.)

$E M\left[p c \mathrm{~cm}^{-6}\right]=\mu_{3} 0.291 d[\mathrm{kpc}] \theta_{G}[\operatorname{arcmin}]\left(N_{e}\left[\mathrm{~cm}^{-3}\right]\right)^{2}$

where $d$ is the adopted distance and the numerical values of the factors $\mu_{1}, \mu_{2}$ and $\mu_{3}$ depend on the particular model chosen for the density distribution of the $\mathrm{H}$ II region. We employed the Gauss model for which $\mu_{1}=0.911, \mu_{2}=3.106, \mu_{3}=1.065$ and $a=1$. The parameter $\theta_{G}$ is the angular size of the source derived assuming a Gaussian brightness temperature distribution for it. This is given by $\theta_{G}=\theta_{s p h} / 1.471$ 


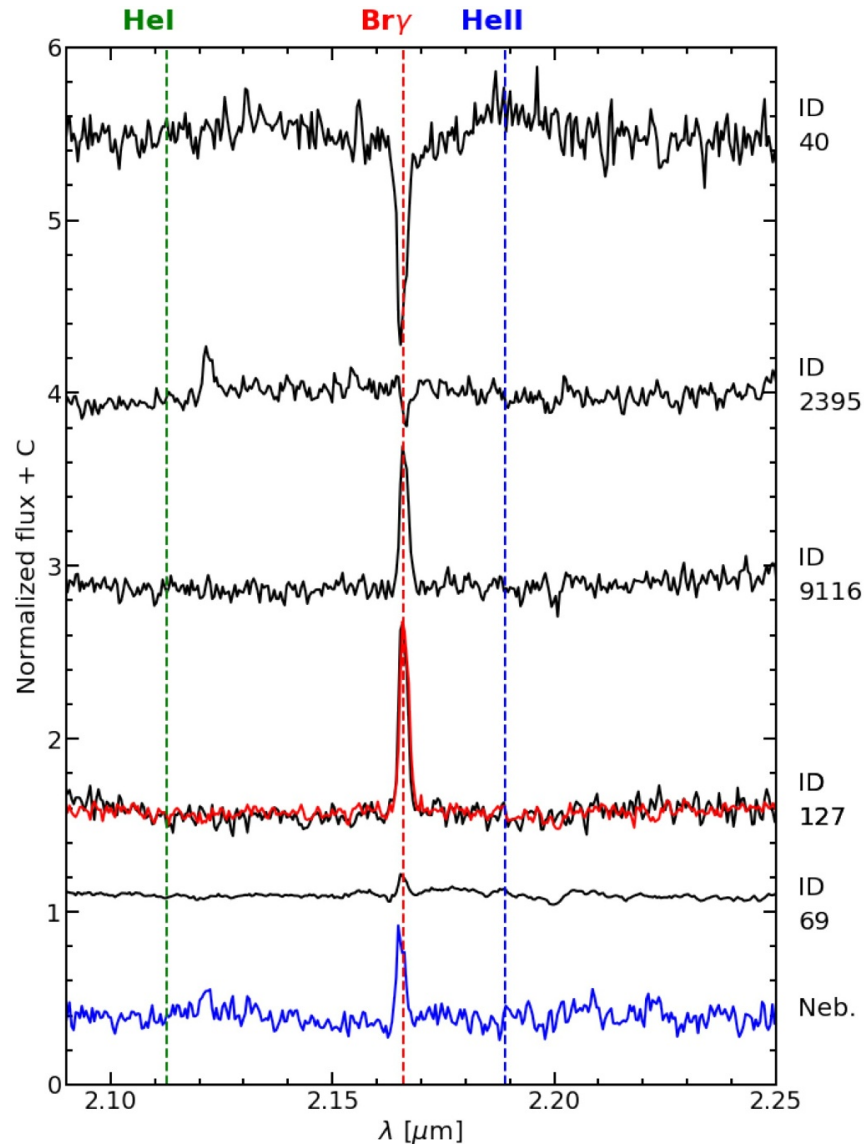

Fig. 5. Stellar and nebular spectra in the DBS 113 region. Several chemical components of each star are indicated with vertical lines. The ID object is indicated on the right and the adopted spectral type of each one is presented in Table 3. Double spectra for ID 127 object correspond to different results for two different slit positions. angular diameter at the adopted distance.

\section{Discussion}

\subsection{IRAS 16571-4029 source}

\subsubsection{Main stellar and ISM parameters}

We performed an identification of several objects in this region (see Section 4 and Fig. 5). All of them could be considered as members of the cluster DBS 113. We also found clues of an abnormal $R_{V}$ value in this direction. In particular, the object identified as ID $127 \equiv 2 \mathrm{MASS}$ J17003386-4033382, classified as O9-B1, was already observed by Bik et al. (2005, 2006) using high dispersion spectroscopy, and they recognized it as a triple system composed by stars identified as 165714029-820 (O8V-B1V), 16571-4029 1610 (O8V-B2.5V) and 16571-4029 1281 (YSO). Our spectroscopic data, with lower spectral resolution, showed the combination of these stars for this object, revealing a strong $\mathrm{Br} \gamma$ emission line. In general, our spectroscopic and photometric analysis at optical/IR bands allowed us to recognize the presence of an important amount of early MS stars (2 O-type and 15 B-type) and 20 objects with IR color excess. Additionally, our study with WISE data revealed 2 Class I, 8 Class II YSO candidates.

The cross-correlation procedure performed among our optical/IR photometric data (ugriJHK) and those data available on several catalogs (2MASS, WISE) yielded stellar energy distributions (SEDs) ranging from 0.3 to $22 \mu \mathrm{m}$ for selected objects in the region of IRAS 16571-4029 source. In this procedure, we used the corresponding zero point fluxes given at NASA / IPAC Infrared Science Archive ${ }^{7}$. The obtained SEDs are presented in Fig. 16 and their comparison showed that adopted MS stars and objects with IR excess (three upper panels) present a maximum at about 1.5-2 $\mu \mathrm{m}$ and then a decreasing behavior. Conversely, it is possible to note that the adopted objects identified as Class I YSO (lower panel, yellow symbols) present a rising energy distribution, whereas objects identified as Class II YSO (lower panel, orange symbols) present a minimum at about 5-6 $\mu \mathrm{m}$ and then a stable or rising shape at longer wavelengths.

Regarding the ISM parameter values for IRAS 16571-4029 source,

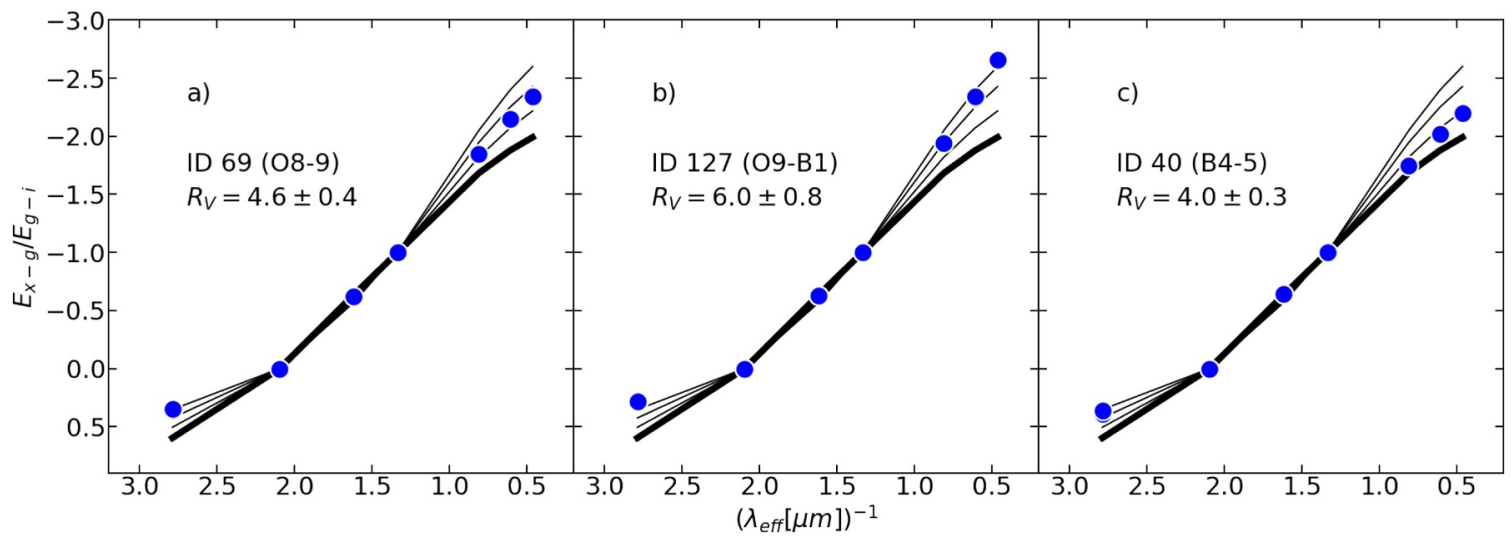

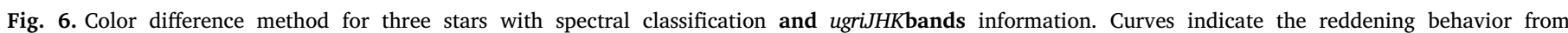
Cardelli et al. (1989) for $R_{V}=3.1,4.0,5.0$ and 6.0 (from the bottom up).

(Mezger and Henderson, 1967)) with $\theta_{s p h}$ obtained as explained in Section 5.1.

We also estimated the "excitation parameter" $(\mu)$ as defined by Wilson et al. (2013). This value is indicative of the number of Lyman photons absorbed by the $\mathrm{HI}$ and generate the HII region. The corresponding equation is:

$\mu\left[p c \mathrm{~cm}^{-2}\right]=R_{S}[p c]\left(N_{e}\left[\mathrm{~cm}^{-3}\right]\right)^{2 / 3}$

where $R_{s}$ is the linear Strömgren radius corresponding to the $\theta_{s p h}$ they were computed following the guidelines given at Sect. 5.2. In particular, $T_{e}$ was already computed by Caswell and Haynes (1987) $\left(T_{e}\right.$ $\simeq 5000 \mathrm{~K})$ and by Quireza et al. (2006b) $\left(T_{e} \simeq 7600 \mathrm{~K}\right)$ from observations at 5 and $8.6 \mathrm{GHz}$ respectively, where the plasma of the region is optically thin. We used both $T_{e}$ values to estimate the ISM parameters and they are presented in Table 5.

\footnotetext{
${ }^{7}$ https://irsa.ipac.caltech.edu
} 

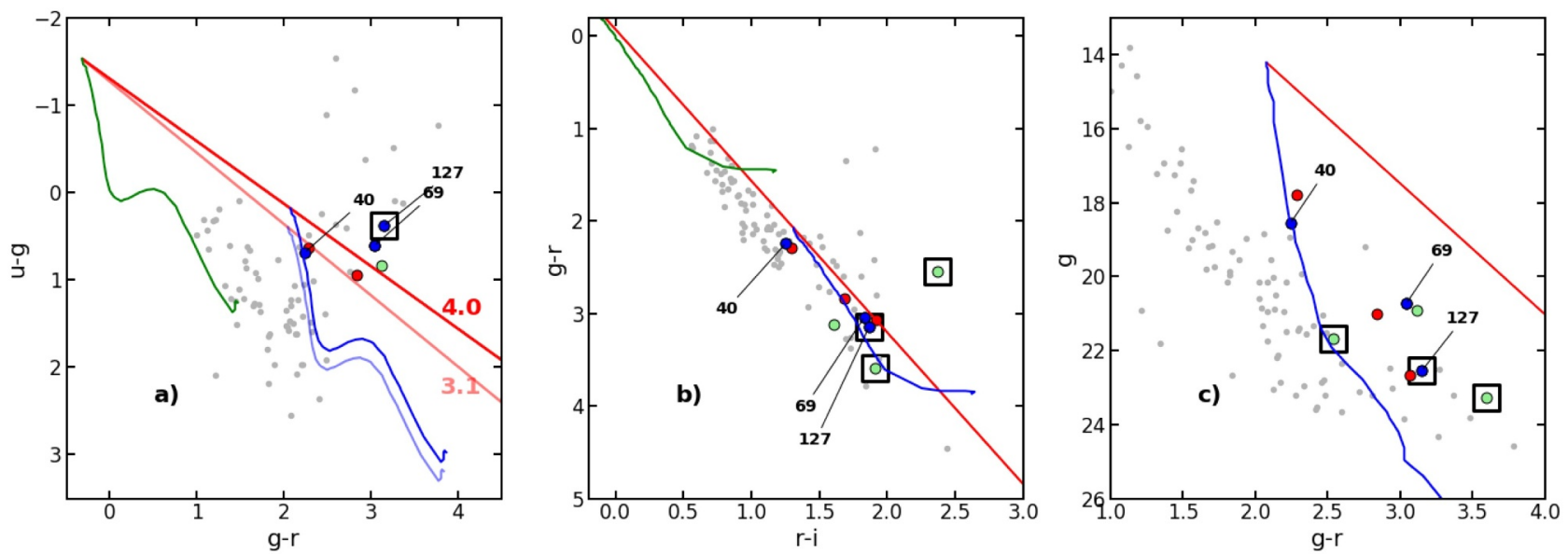

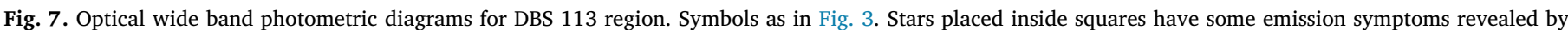

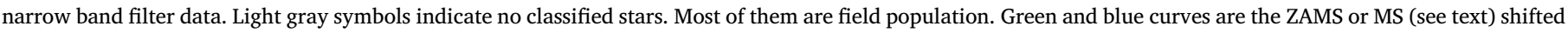

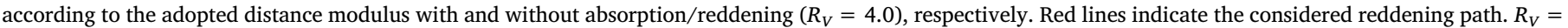

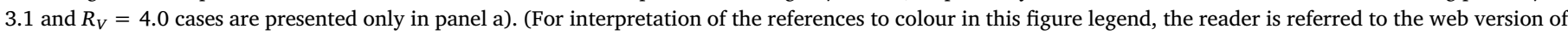
this article.)
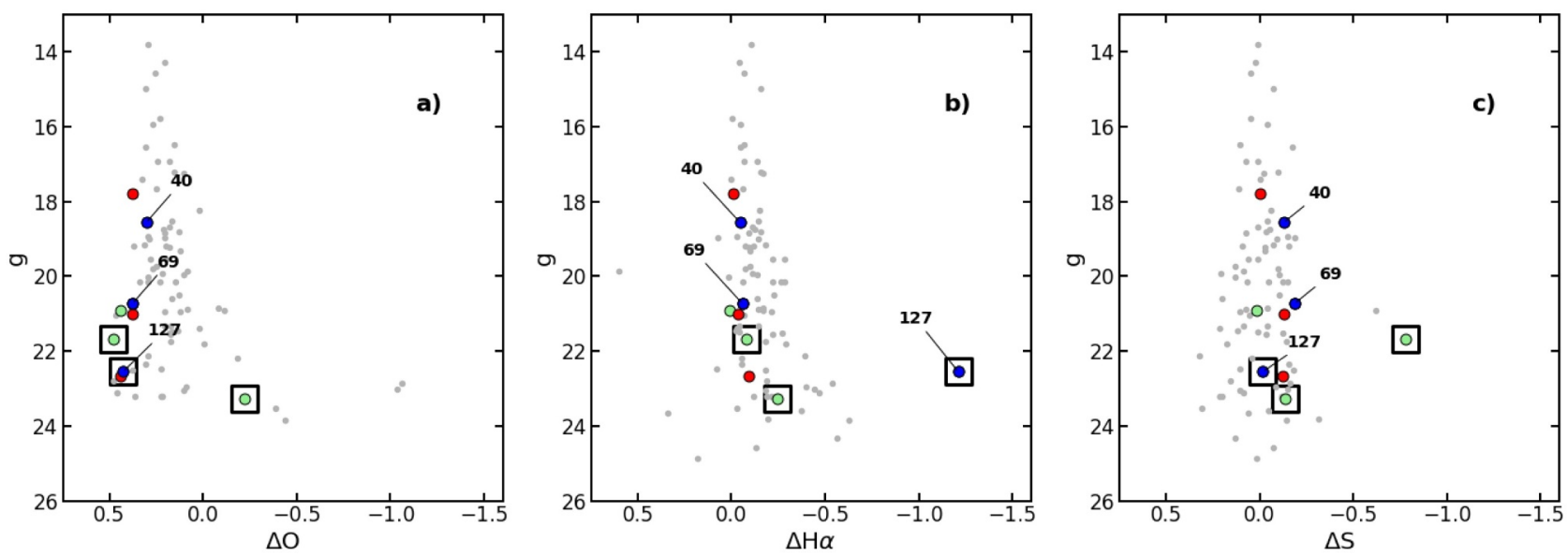

Fig. 8. Narrow band index diagrams for DBS 113 region. Symbols and lines as in Fig. 7.
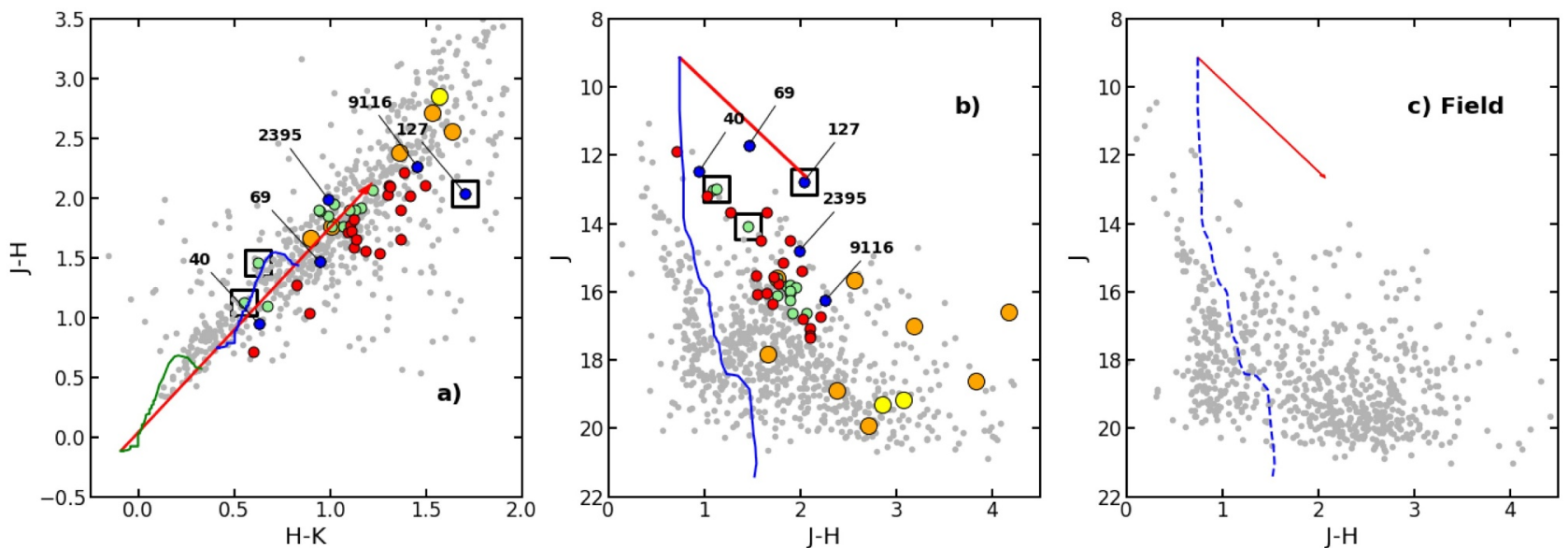

Fig. 9. IR photometric diagrams for DBS 113 and its comparison field region. Symbols and lines as in Fig. 7.

\subsubsection{Distance to the source}

Since we had a rough spectroscopic classification and GAIA astrometric data for several stars in the region, it was possible to estimate spectrophotometric and trigonometric distances. Additionally, the SGPS HI data allowed us to obtain a kinematic distance.
To understand more precisely the HI kinematic behavior in this direction, we obtained the $\mathrm{H}$ I emission and absorption profiles performing the disjoin of the Galactic background emission to the small scale structures around and on the compact H II region. This task was done using a similar procedure to that given by Kothes and 

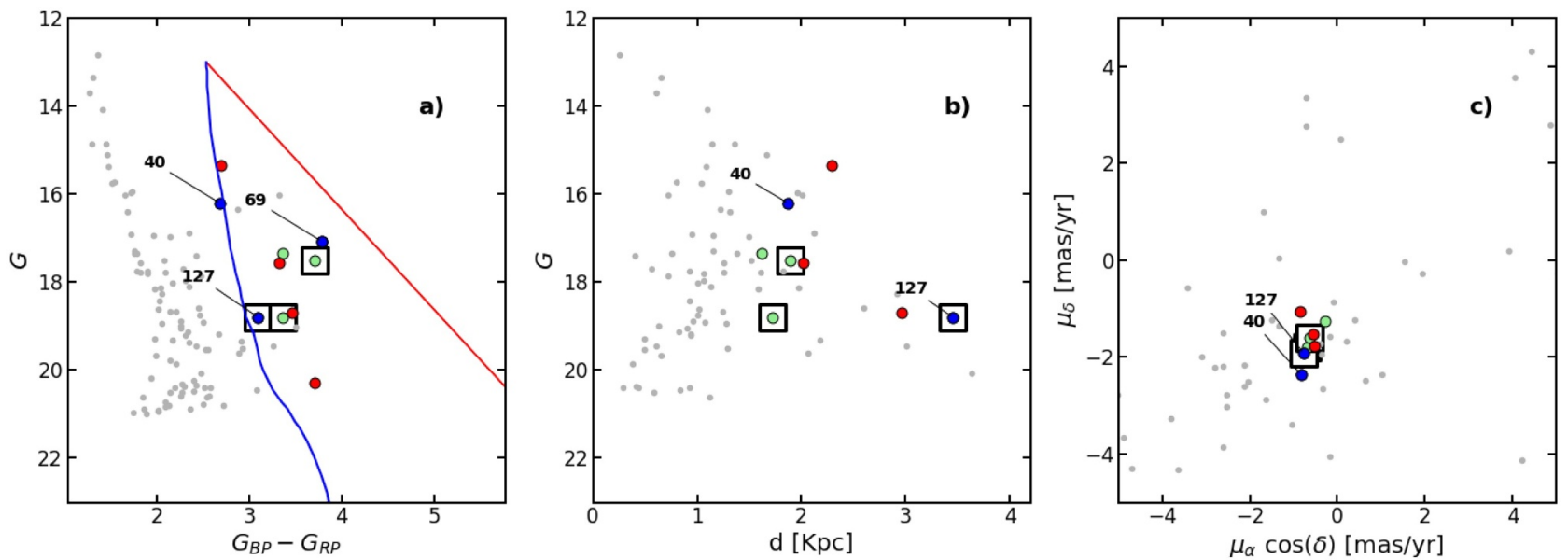

Fig. 10. Photometric and astrometric diagrams for DBS 113 region from GAIA data. Symbols and lines as in Fig. 7.
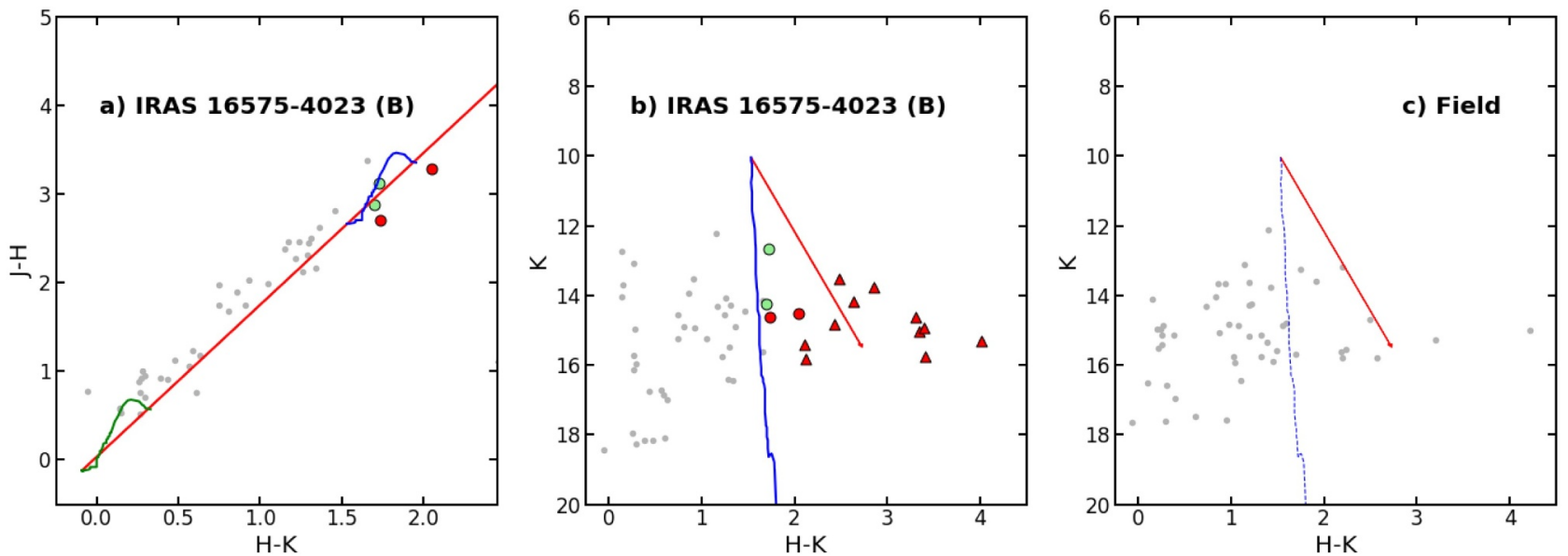

Fig. 11. IR photometric diagrams for IRAS 16575-4023 source (region B) and a comparison field (annulus around region B). Symbols as in Fig. 7.

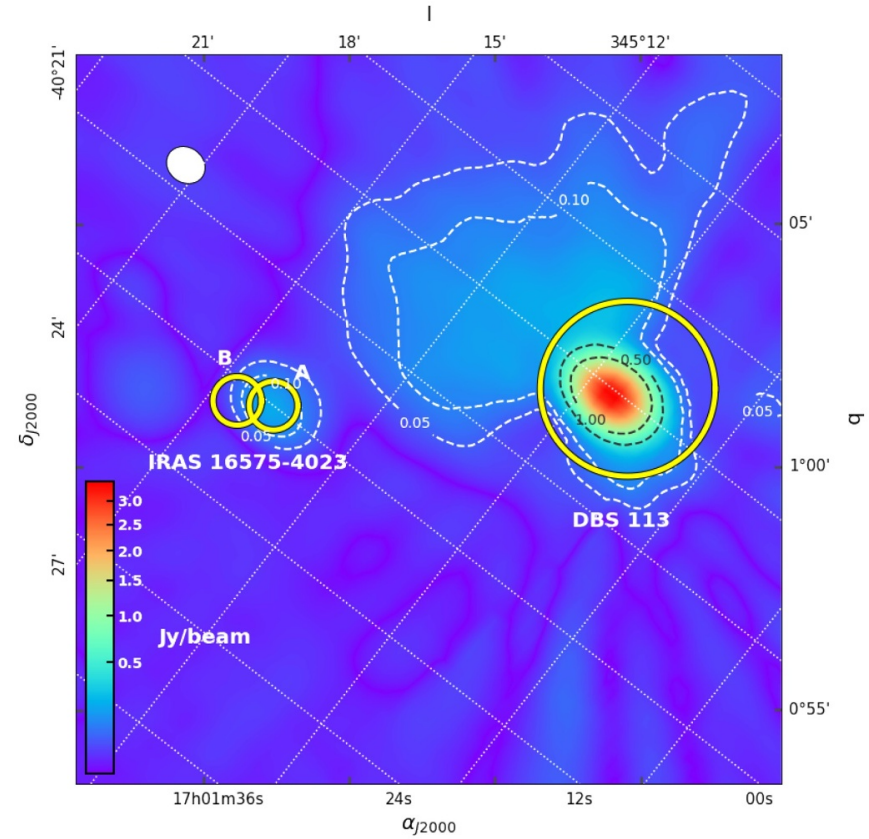

Fig. 12. Radio continuum SUMSS observations at $843 \mathrm{MHz}$. Contour levels at $0.05,0.10,0.50$ and $1.00 \mathrm{Jy}^{-1}$ beam $^{-1}$ are shown. White ellipse drawn in the upper left-hand corner indicates the instrumental beam size. Yellow circles have the same meaning as in Fig. 1.

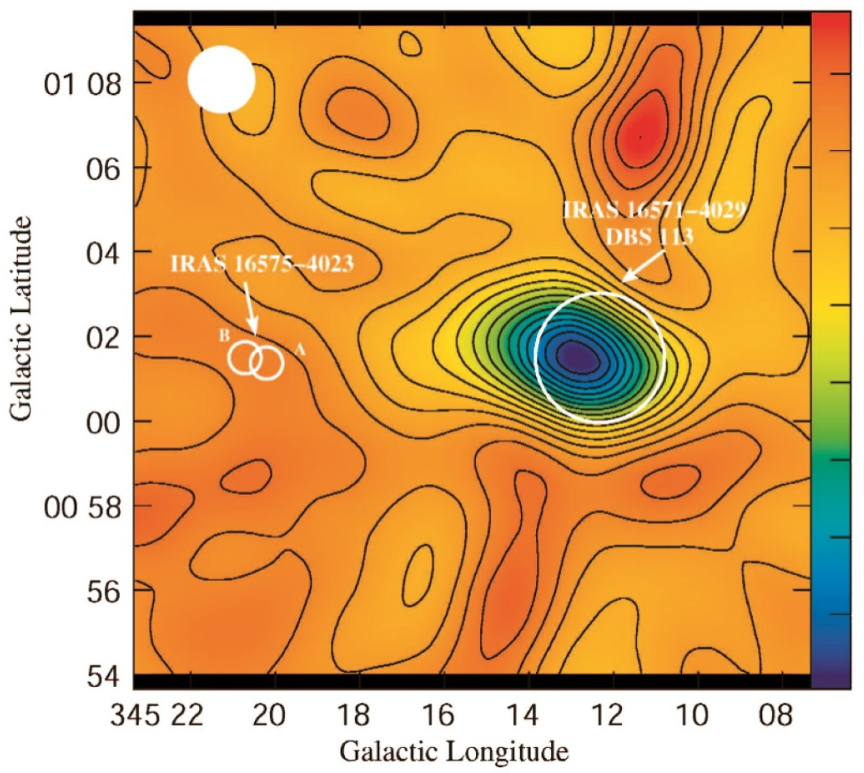

Fig. 13. Average map of $\mathrm{HI}$ obtained with 41 radial velocity maps of the studied area (see text). Contours and colors indicate different brightness temperature values $\left(T_{b}\right)$ following right bar scale. Contours vary from 5 to $100 \mathrm{~K}$ with a step of $5 \mathrm{~K}$. White solid circle drawn in the upper left-hand corner indicates the instrumental beam size. Hollow circles have the same meaning as in Figs. 1 and 12. 

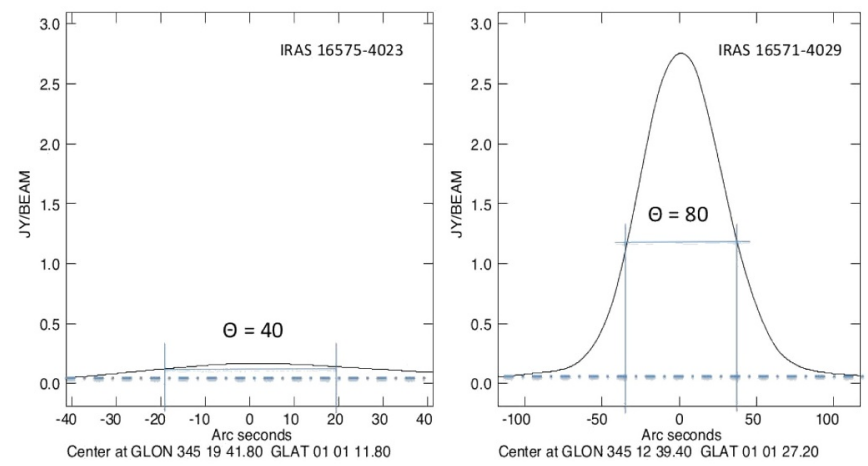

Fig. 14. Flux density profiles from the SUMSS observations at $843 \mathrm{MHz}$ at each IRAS source location. Dashed-point lines represent the adopted background levels. The HPBW of each profile is indicated.

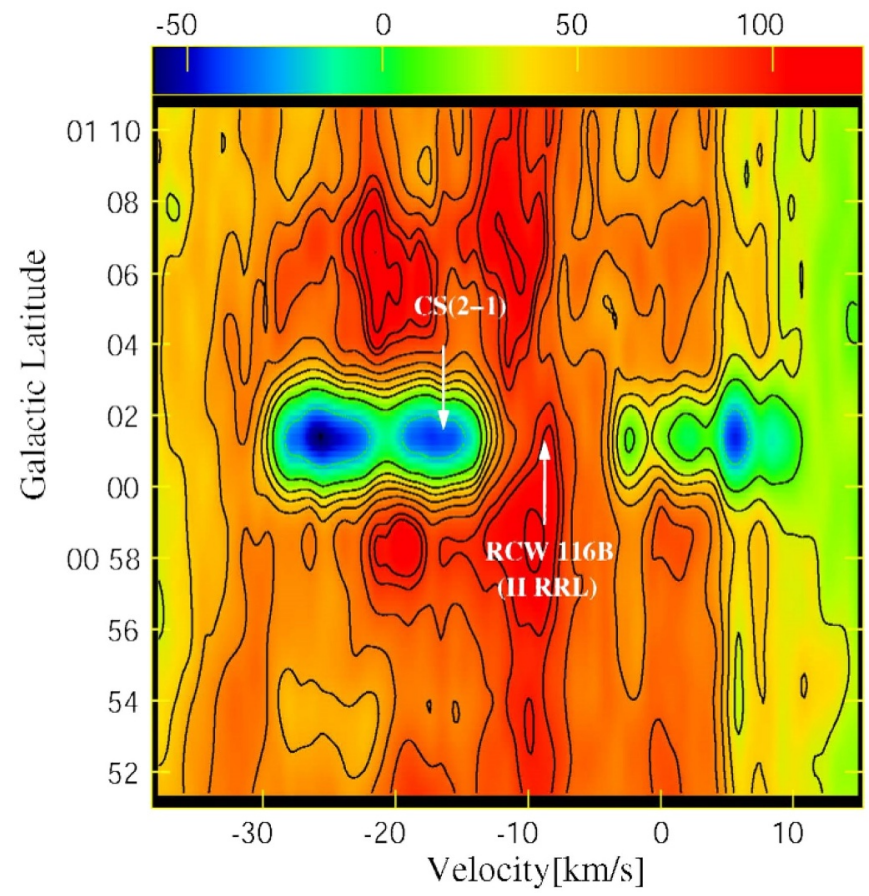

Fig. 15. Position - velocity image of the HI distribution at $21 \mathrm{~cm}\left(l=345.2^{\circ}\right)$. The $T_{b}$ values are indicated with the color scale. Contours and colors indicate different $T_{b}$ values following upper bar scale. The contours are indicated with a step of $12 \mathrm{~K}$. The location of RCW 116B hydrogen RRL at $-9.4 \mathrm{~km} \mathrm{~s}^{-1}$ and CS(21) line at $-15.6 \mathrm{~km} \mathrm{~s}^{-1}$ are also shown.

Kerton (2002). Basically, we convolved each velocity channel of the H I data cube with a gaussian beam of FWHM $=10$ ' to obtain the emission profiles and the subtraction between the original data and its smoothed version to get the absorption profiles. The resulting profiles in the direction of the absorption minimum near the IRAS 16571-4029 source are presented in Fig. 17a. This figure clearly shows the location of the tangential velocity $\left(V_{T}=-150 \mathrm{~km} \mathrm{~s}^{-1}\right)$ at the emission feature with the largest negative velocity value, and the presence of two main absorption features with an upper velocity value of $\sim-30 \mathrm{~km} \mathrm{~s}^{-1}$. In particular, the fact that there are no absorption features up to the tangential velocity reveals they are caused closer than the tangential point in this direction.

A roughly and traditional analysis of Figs. 15 and 17 indicate that the corresponding radial velocity value of the studied $\mathrm{H}$ II region is given by the highest negative value of the absorption feature ( $-30 \mathrm{~km} \mathrm{~s}^{-1}$ ). However, this value is inconsistent with those given in previous studies of this region at radio wavelength. A compilation of the
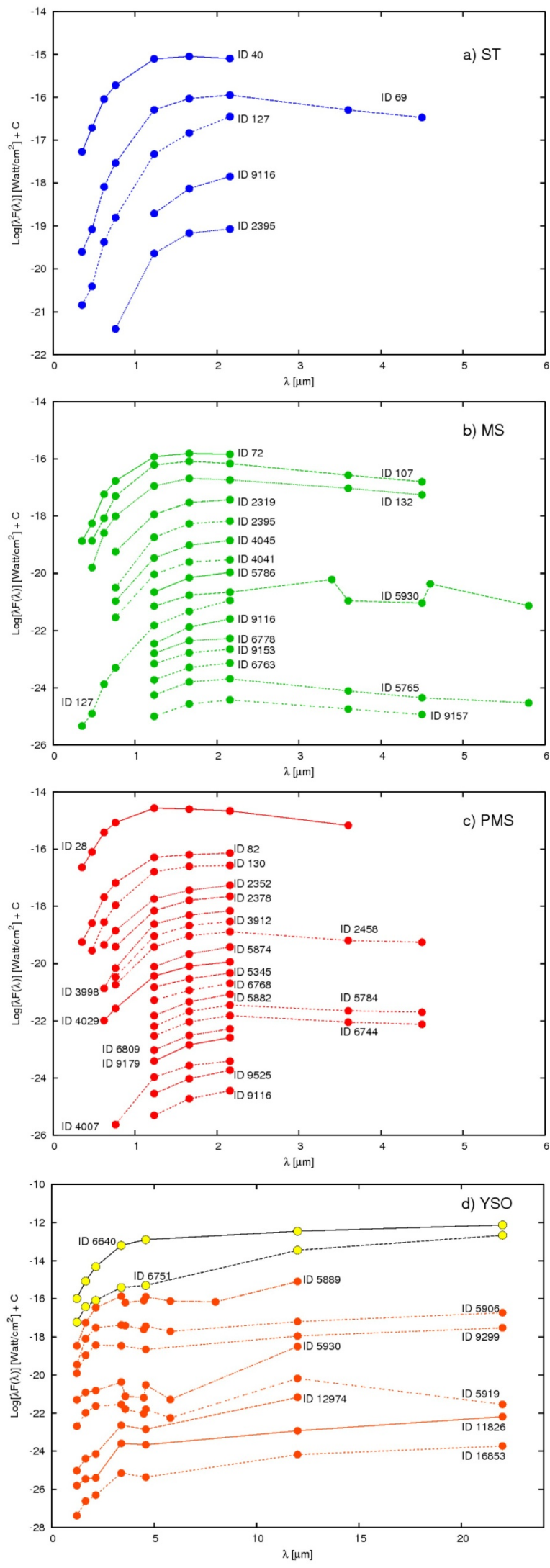

Fig. 16. The SEDs of selected objects considered members of DBS 113. Symbols along the panels have the same meaning as in Fig. 7. 

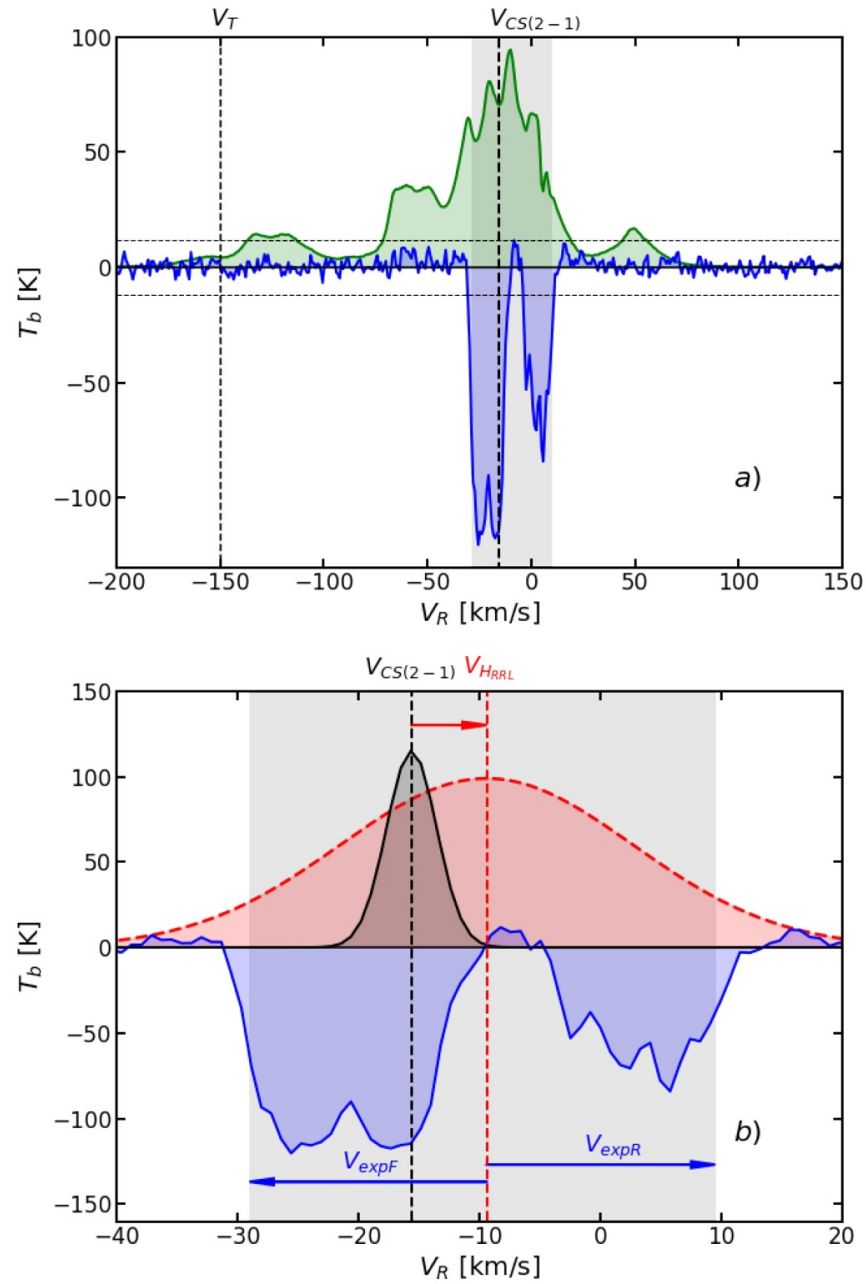

Fig. 17. a) Emission (green) and absorption (blue) HI profiles at the center of the RCW 116B region covering almost all the velocity channels. Vertical dashed lines indicate the tangential velocity $\left(V_{T}=-150 \mathrm{~km} \mathrm{~s}^{-1}\right)$ and the CS molecular line $\left(V_{C S}=-15.6 \mathrm{~km} \mathrm{~s} \mathrm{~s}^{-1}\right)$. The horizontal lines represent $\Delta T_{b}=12 \mathrm{~K} \sim 5 \mathrm{rms}_{b k g}$ b) Absorption (blue) profile together with a schematic representation of the CS lines (black fill curve) and HI RRL (dashed red curve) as two gaussian profiles with arbitrary height. Shadow regions in both panels indicate the velocity range for absorption features $\left(-29 \mathrm{~km} \mathrm{~s}^{-1}<V_{R}<\right.$ $+9 \mathrm{~km} \mathrm{~s}^{-1}$ ). See text for arrows meaning. (For interpretation of the references to colour in this figure legend, the reader is referred to the web version of this article.)

Table 6

Compilation of kinematic information in the direction of IRAS 16571-4029 $\equiv$ G345.23+1.03 source.

\begin{tabular}{|c|c|c|c|}
\hline Line & $\begin{array}{l}V_{R_{L S R}} \\
{\left[\mathrm{~km} \mathrm{~s}^{-1}\right]}\end{array}$ & $\begin{array}{l}\Delta V \\
{\left[\mathrm{~km} \mathrm{~s}^{-1}\right]}\end{array}$ & Ref. \\
\hline H109 $\alpha$ & $-9.40 \pm 1.80$ & $28.50 \pm 3.60$ & $1 ; 2$ \\
\hline $\mathrm{H}$ & $-11.54 \pm 0.13$ & $29.11 \pm 0.39$ & 4 \\
\hline${ }^{4} \mathrm{He}$ & $-11.26 \pm 0.39$ & $21.69 \pm 1.43$ & 4 \\
\hline $\operatorname{CS}(2-1)$ & $-15.60 \pm 0.52$ & $4.90 \pm 0.52$ & 3 \\
\hline $\operatorname{CS}(7-6)$ & $-15.03 \pm 0.03$ & $3.12 \pm 0.07$ & 5 \\
\hline CII & $-16.20 \pm 0.51$ & $4.44 \pm 1.18$ & 4 \\
\hline $\mathrm{HCN}(4-3)$ & $-14.69 \pm 0.12$ & $5.65 \pm 0.41$ & 5 \\
\hline
\end{tabular}

Note:(1) Wilson et al. (1970); (2) Caswell and Haynes (1987); (3) Bronfman et al. (1996) and adopted errors correspond to the velocity resolution; (4) Quireza et al. (2006a); (5) Liu et al. (2016) velocity values are presented in Table 6 , which highlights the difference in velocity values between those obtained from hydrogen radio recombination lines $\left(\sim-9\right.$ and $\sim-11 \mathrm{~km} \mathrm{~s}^{-1}$ with $\left.\Delta V \sim 30 \mathrm{~km} \mathrm{~s}^{-1}\right)$ and those resulting from molecular studies $\left(\sim-15 \mathrm{~km} \mathrm{~s}^{-1}\right.$ with $\Delta V \sim 5 \mathrm{~km} \mathrm{~s}^{-1}$ ). Therefore, Fig. 15 shows an absorption component up to $\sim 20 \mathrm{~km} \mathrm{~s}^{-1}$ beyond the hydrogen RRL velocity. Using the Galactic rotation model of Fich et al. (1989) and adopting the corresponding nearest distances, the presented $V_{R}$ values: $-9,-11,-15$ and $-30 \mathrm{~km} \mathrm{~s}^{-1}$ yielded respectively kinematic distance values of 1.25, 1.45, 1.95 and $3.2 \mathrm{kpc}$. These discrepancies could be understood as a consequence of local departures from the rotation curve. It is not strange since we are working at Galactic longitude values close to the Galactic center direction and we could also be dealing with somewhat turbulent flows (Jones and Dickey, 2012). Besides, the possible presence of streaming motions could induce local kinematic distance ambiguities (Fish et al., 2003).

Under these circumstances, the kinematic analysis did not seem to provide a reliable way to estimate the distance to the IRAS 16571-4029 source and we adopted the value computed from GAIA data which is consistent with the spectrophotometric one (see Table 4). Additionally, with this distance value, the kinematic data could be reanalyzed taking into account that the $\Delta V$ value of $\sim 28 \mathrm{~km} \mathrm{~s}^{-1}$ indicated by Wilson et al. (1970) is comparable with the complete extension, from +10 to $-30 \mathrm{~km} \mathrm{~s}^{-1}$, of the minimum presented in Fig. 15. These values could be understood as due to turbulent movements and/or a hydrogen expanding shell $\left(V_{\text {Rexp }} \sim V_{\text {Fexp }} \sim 20 \mathrm{~km} \mathrm{~s}^{-1}\right)$ around a central value of $\sim-10 \mathrm{~km} \mathrm{~s}^{-1}$ given by the HI RRLs, and this would be shifted by a peculiar velocity of about $5 \mathrm{~km} \mathrm{~s}^{-1}$ from the $\sim-15 \mathrm{~km} \mathrm{~s}^{-1}$ given by the molecular cloud velocity measurements that correspond with a kinematic distance near to $2 \mathrm{kpc}$ (see Fig. 17b for a schematic representation of this scenario). These kind of behavior was already found on other HII regions by Caswell and Haynes (1987) and Kothes and Kerton (2002). In fact, Morales et al. (2013) already identified DBS 113 as a partially embedded cluster since the peak at the submillimetric observations is significantly shifted in location from the cluster itself, supporting the idea of a cluster with a kinematic behavior different to that one of the molecular cloud.

\subsubsection{Energetic balance in the source}

The estimated ISM parameters for the IRAS 16571-4029 source revealed it as a compact or classical HII region. Following Kurtz et al. (1994), the ionization-bounded case is probably a very good assumption but it is not a dust-free nebula. Therefore, it is possible to compare the energy needed to excite the HII region with that provided by the brightest stars of DBS 113 from the values found for the excitation parameter ( $\mu$, see Section 5.2) and the ionization parameter ( $U$, see Section 4.3). The ratio $U / \mu \approx 1.7>1$ indicated that cluster stars produce enough radiant energy to generate the HII region. Since $U \propto N_{L y \text { stars }}^{1 / 3}$ then:

$\frac{U}{\mu}=\left(\frac{N_{L y ~ s t a r s}}{N_{L y H I I}}\right)^{1 / 3}$

where $N_{L y H I I}$ indicates the amount of Lyman photons required to generate the observed HII region, resulting to be $N_{L y H I I} \sim 3.1-3.810^{48}$ photons $\mathrm{s}^{-1}$. Therefore, there are $\sim 70-75 \%$ of the stellar Lyman photons $\left(1-(\mu / U)^{3}\right)$ heating the surrounding dust. It includes both the ISM dust component and the probable circumstellar ones (Kurtz et al., 1994).

\subsection{IRAS 16575-4023 source}

This IRAS source is a very poor object and there is almost no information about it. The source was only identified as an HII region according to Bronfman et al. (1996), and Volk et al. (1991) obtained a unique and unusual IR spectrum with a possible absorption at $11.3 \mu \mathrm{m}$. 
Table 7

OB candidate objects in the region of IRAS 16575-4023 (B) source .

\begin{tabular}{|c|c|c|c|c|c|c|c|c|c|c|}
\hline$I D$ & 2MASS & $\alpha_{J 2000}$ & $\delta_{J 2000}$ & $J$ & & $H$ & & K & & \\
\hline 7992 & $17010017-4027289$ & $17: 01: 00.2$ & $-40: 27: 28.9$ & 17.16 & \pm- & 15.99 & $\pm \quad 0.02$ & 13.52 & \pm & 0.02 \\
\hline 8061 & $17010073-4027449$ & $17: 01: 01.0$ & $-40: 27: 44.9$ & 18.84 & $\pm \quad 0.07$ & 15.95 & 0.02 & 14.26 & \pm & 0.02 \\
\hline 8064 & $17010081-4027293$ & $17: 01: 00.8$ & $-40: 27: 29.4$ & 19.84 & \pm 0.12 & 16.56 & \pm 0.03 & 14.51 & \pm & 0.03 \\
\hline 8110 & $17010114-4027483$ & $17: 01: 01.2$ & $-40: 27: 48.1$ & 17.51 & \pm 0.02 & 14.39 & \pm 0.02 & 12.66 & \pm & 0.02 \\
\hline 8117 & $17010143-4027269$ & $17: 01: 01.4$ & $-40: 27: 27.0$ & 19.08 & \pm 0.06 & 16.37 & 0.02 & 14.63 & \pm & 0.02 \\
\hline 8122 & $17010016-4027212$ & $17: 01: 00.2$ & $-40: 27: 21.2$ & 18.16 & $\pm \quad-$ & 16.64 & \pm 0.03 & 13.78 & \pm & 0.04 \\
\hline
\end{tabular}

Note: A complete version of this table including all the studied photometric bands is available on-line at the CDS.

As was indicated before, to study this source we selected two regions: A and B. The stellar population in region A presents an overdensity of faint stars which does not seem related with the IRAS source. However, in this location the radio continuum SUMSS observations at $843 \mathrm{MHz}$ reach their peak. Conversely, region B revealed a spatial concentration of very faint and red objects consistent with high reddened early type stars (OB; see CMDs in Fig. 11 and Table 7) located behind the molecular cloud AGAL G345.336+01.021. The location of these OB stars candidates is shown in the PACS / Herschel image (see Fig. 4b) as the center of a clear bubble of about 2' in radius reinforcing the presence of early stars. The adopted distance for these stars ( $\sim 2 \mathrm{kpc}$ ) agrees with the kinematic one of $1.9 \mathrm{kpc}$ indicated by Wienen et al. (2015) for the molecular cloud. Additionally, the research of CS(2-1) lines done by Bronfman et al. (1996) in this direction provided a value of $V_{R}(\mathrm{LSR})=$ $-14.5 \mathrm{~km} \mathrm{~s}^{-1}$ with a $\Delta V=2.8 \mathrm{~km} \mathrm{~s}^{-1}$. By using the Galactic rotation model of Fich et al. (1989), and adopting the nearest distance, we could also estimate a value of $d=1.9 \mathrm{kpc}$.

\section{Conclusions}

We studied the IRAS 16571-4029 and 16575-4023 sources located in the fourth Galactic quadrant of the Milky Way plane through a multiwavelength and multi-technique approach. We focused our attention on embedded cluster DBS 113 associated with IRAS 16571-4029 since this region appears as more relevant and there were contradictions about its distance. We used then several methods to estimate this value and we found the most probable one is $2 \mathrm{kpc}$. This value is consistent with previous estimations based on studies of the associated molecular cloud G345.5 + 1.0 and located this star formation region at the inner side of the Sagittarius-Carina Galactic arm. This distance value allowed us to obtain more reliable estimations of the physical parameters of the region. From the relationship studied between $U$ and $\mu$ parameters it is possible to conclude that the region is limited by density, and about $70-75 \%$ of Lyman photons produced by the early stars are consumed by the dust. Additionally, our kinematic study at this direction revealed a particular behavior of the gas in this part of the Galaxy, showing much larger radial velocity values (in absolute terms) at the location of the cluster and HII region than those expected from the usual rotation model.

It must be noticed the importance to use stellar spectroscopic classification along with $u$ band data to obtain a better estimation of the reddening law for this cluster. Our photometric and spectroscopic analysis using optical/IR bands was useful to identify early stars and sources with IR excess in this region. Additionally, by using WISE FIR data we could classify several objects as YSOs.

Regarding IRAS 16575-4023 source, we found that the brightest star in the region is a field foreground one according to GAIA DR2 data, and we identified a probably very faint and reddened young stellar population responsible for the HII region and an IR bubble was identified at 70 and $160 \mu \mathrm{m}$ bands. The discovered young stellar candidates deserve a future spectroscopic follow up to confirm their nature.

\section{Acknowledgements}

G.B and M.A.C. acknowledge support from CONICET (PIPs 112-
201701-00055 and 112-201701-00507). S.R.A. acknowledges the support by the FONDECYT Iniciación project No 11171025 and the CONICYT PAI "Concurso Nacional Inserción de Capital Humano Avanzado en la Academia 2017" project PAI 79170089. Support for J.B. is provided by the Ministry of Economy, Development, and Tourism's Millennium Science Initiative through grant IN 120009, awarded to The Millennium Institute of Astrophysics, The authors are much obliged for the use of the NASA Astrophysics Data System, of the SIMBAD database and ALADIN tools (CDS, France). This publication was based on: a) Observations and data products from observations made with ESO Telescopes at La Silla or Paranal Observatories; b) Observations obtained at the Gemini Observatory, which is operated by the Association of Universities for Research in Astronomy, Inc., under a cooperative agreement with the NSF on behalf of the Gemini partnership: the NSF (USA), NRC (Canada), CONICYT (Chile), Sec. de Ciencia, Tecnología e Innovación Productiva (Argentina), Min. da Ciência, Tecnologia e Inovação (Brazil), and KASSI (Republic of Korea); c) The 2MASS, which is a joint project of the University of Massachusetts and the IPAC/ California Institute of Technology, funded by the NASA and the NSF; d) Data products from the WISE, which is a joint project of the University of California, Los Angeles, and the JPL/California Institute of Technology, and is funded bythe NASA; e) Data from the ESA mission Gaia, processed by the Gaia DPAC. Funding for the DPAC has been provided by national institutions, in particular the institutions participating in the Gaia Multilateral Agreement; f) The SGPS is a project with images obtained at high resolution using the Australia Telescope Compact Array and the Parkes Radio Telescope; g) The SUMSS is a radio imaging survey carried out with the Molonglo Observatory Synthesis Telescope (MOST), Australia. We would like to thank the English Department at FCAG (UNLP) for proofreading this paper. We also wish to thank our referee suggestions and comments, which improved the original version of this work.

\section{Author statement}

G. Baume: - Photometric data

M.A.Corti:- Radio data

J. Borissova and S. Ramirez Alegria: - Spectroscopic data

\section{CRediT authorship contribution statement}

G. Baume: Conceptualization, Data curation, Methodology, Investigation, Visualization. M.A. Corti: Conceptualization, Data curation, Methodology, Investigation. J. Borissova: Investigation. S. Ramirez Alegria: Investigation. A.V. Corvera: Writing - review \& editing, Investigation.

\section{Declaration of Competing Interest}

The authors have not any financial and personal relationships with other people or organizations that could inappropriately influence (bias) their work Note: The web link for the "Declaration of Interest template" was not available 


\section{References}

Bailer-Jones, C.A.L., 2015. PASP 127, 994. https://doi.org/10.1086/683116.

Baume, G., Carraro, G., Momany, Y., 2009. MNRAS 398, 221-232. https://doi.org/10. 1111/j.1365-2966.2009.14934.x.

Bik, A., Kaper, L., Hanson, M.M., Smits, M., 2005. A\&A 440, 121-137. https://doi.org/10. 1051/0004-6361:20042597.

Bik, A., Kaper, L., Waters, L.B.F.M., 2006. A\&A 455, 561-576. https://doi.org/10.1051/ 0004-6361:20042403.

Bonnarel, F., Fernique, P., Bienaymé, O., Egret, D., Genova, F., Louys, M., Ochsenbein, F., Wenger, M., Bartlett, J.G., 2000. A\&AS 143, 33-40. https://doi.org/10.1051/ aas: 2000331 .

Borissova, J., Ivanov, V.D., Hanson, M.M., Georgiev, L., Minniti, D., Kurtev, R., Geisler, D., 2008. A\&A 488, 151-159. https://doi.org/10.1051/0004-6361:20079255.

Bronfman, L., Nyman, L.-A., May, J., 1996. A\&AS 115, 81.

Brown, A.G.A., Vallenari, A., Prusti, T., de Bruijne, J.H.J., Babusiaux, C., Bailer-Jones, C.A.L., Biermann, M., Evans, D.W., Eyer, L., Jansen, F., Jordi, C., Klioner, S.A., Lammers, U., Lindegren, L., Luri, X., Mignard, F., Panem, C., Pourbaix, D., Randich, S., Sartoretti, P., Siddiqui, H.I., Soubiran, C., van Leeuwen, F., Walton, N.A., Arenou, F., Bastian, U., Cropper, M., Drimmel, R., Katz, D., Lattanzi, M.G., Bakker, J., Cacciari, C., Castañeda, J., Chaoul, L., Cheek, N., De Angeli, F., Fabricius, C., Guerra, R., Holl, B., Masana, E., Messineo, R., Mowlavi, N., Nienartowicz, K., Panuzzo, P., Portell, J., Riello, M., Seabroke, G.M., Tanga, P., Thévenin, F., Gracia-Abril, G., Comoretto, G., Garcia-Reinaldos, M., Teyssier, D., Altmann, M., Andrae, R., Audard, M., Bellas-Velidis, I., Benson, K., Berthier, J., Blomme, R., Burgess, P., Busso, G., Carry, B., Cellino, A., Clementini, G., Clotet, M., Creevey, O., Davidson, M., De Ridder, J., Delchambre, L., Dell'Oro, A., Ducourant, C., Fernández-Hernández, J. Fouesneau, M., Frémat, Y., Galluccio, L., García-Torres, M., González-Núñez, J., González- Vidal, J.J., Gosset, E., Guy, L.P., Halbwachs, J.L., Hambly, N.C., Harrison, D.L., Hernández, J., Hestroffer, D., Hodgkin, S.T., Hutton, A., Jasniewicz, G., JeanAntoine- Piccolo, A., Jordan, S., Korn, A.J., Krone- Martins, A., Lanzafame, A.C., Lebzelter, T., Löffler, W., Manteiga, M., Marrese, P.M., Martín-Fleitas, J.M., Moitinho, A., Mora, A., Muinonen, K., Osinde, J., Pancino, E., Pauwels, T., Petit, J.M., RecioBlanco, A., Richards, P.J., Rimoldini, L., Robin, A.C., Sarro, L.M., Siopis, C., Smith, M., Sozzetti, A., Süveges, M., Torra, J., van Reeven, W., Abbas, U., Abreu Aramburu, A., Accart, S., Aerts, C., Altavilla, G., Álvarez, M.A., Alvarez, R., Alves, J., Anderson, R.I., Andrei, A.H., Anglada Varela, E., Antiche, E., Antoja, T., Arcay, B., Astraatmadja, T.L., Bach, N., Baker, S.G., Balaguer-Núñez, L., Balm, P., Barache, C., Barata, C., Barbato, D., Barblan, F., Barklem, P.S., Barrado, D., Barros, M., Barstow, M.A., Bartholomé Muñoz, S., Bassilana, J.L., Becciani, U., Bellazzini, M., Berihuete, A., Bertone, S., Bianchi, L., Bienaymé, O., Blanco-Cuaresma, S., Boch, T., Boeche, C., Bombrun, A., Borrachero, R., Bossini, D., Bouquillon, S., Bourda, G., Bragaglia, A. Bramante, L., Breddels, M.A., Bressan, A., Brouillet, N., Brüsemeister, T., Brugaletta, E., Bucciarelli, B., Burlacu, A., Busonero, D., Butkevich, A.G., Buzzi, R., Caffau, E., Cancelliere, R., Cannizzaro, G., Cantat-Gaudin, T., Carballo, R., Carlucci, T., Carrasco, J.M., Casamiquela, L., Castellani, M., Castro-Ginard, A., Charlot, P., Chemin, L., Chiavassa, A., Cocozza, G., Costigan, G., Cowell, S., Crifo, F., Crosta, M., Crowley, C., Cuypers, J., Dafonte, C., Damerdji, Y., Dapergolas, A., David, P., David, M., de Laverny, P., De Luise, F., De March, R., de Martino, D., de Souza, R., de Torres, A. Debosscher, J., del Pozo, E., Delbo, M., Delgado, A., Delgado, H.E., Di Matteo, P., Diakite, S., Diener, C., Distefano, E., Dolding, C., Drazinos, P., Durán, J., Edvardsson, B., Enke, H., Eriksson, K., Esquej, P., Eynard Bontemps, G., Fabre, C., Fabrizio, M., Faigler, S., Falcão, A.J., Farràs Casas, M., Federici, L., Fedorets, G., Fernique, P., Figueras, F., Filippi, F., Findeisen, K., Fonti, A., Fraile, E., Fraser, M., Frézouls, B., Gai, M., Galleti, S., Garabato, D., García-Sedano, F., Garofalo, A., Garralda, N., Gavel, A., Gavras, P., Gerssen, J., Geyer, R., Giacobbe, P., Gilmore, G., Girona, S., Giuffrida, G., Glass, F., Gomes, M., Granvik, M., Gueguen, A., Guerrier, A., Guiraud, J., GutiérrezSánchez, R., Haigron, R., Hatzidimitriou, D., Hauser, M., Haywood, M., Heiter, U., Helmi, A., Heu, J., Hilger, T., Hobbs, D., Hofmann, W., Holland, G., Huckle, H.E., Hypki, A., Icardi, V., Janßen, K., Jevardat de Fombelle, G., Jonker, P.G., Juhász, Á.L., Julbe, F., Karampelas, A., Kewley, A., Klar, J., Kochoska, A., Kohley, R., Kolenberg, K., Kontizas, M., Kontizas, E., Koposov, S.E., Kordopatis, G., Kostrzewa-Rutkowska, Z., Koubsky, P., Lambert, S., Lanza, A.F., Lasne, Y., Lavigne, J.B., Le Fustec, Y., Le Poncin-Lafitte, C., Lebreton, Y., Leccia, S., Leclerc, N., Lecoeur-Taibi, I., Lenhardt, H., Leroux, F., Liao, S., Licata, E., Lindstrøm, H.E.P., Lister, T.A., Livanou, E., Lobel, A., López, M., Managau, S., Mann, R.G., Mantelet, G., Marchal, O., Marchant, J.M., Marconi, M., Marinoni, S., Marschalkó, G., Marshall, D.J., Martino, M., Marton, G., Mary, N., Massari, D., Matijevič, G., Mazeh, T., McMillan, P.J., Messina, S., Michalik, D., Millar, N.R., Molina, D., Molinaro, R., Molnár, L., Montegriffo, P., Mor, R., Morbidelli, R., Morel, T., Morris, D., Mulone, A.F., Muraveva, T., Musella, I., Nelemans, G., Nicastro, L., Noval, L., O’Mullane, W., Ordénovic, C., Ordóñez-Blanco, D., Osborne, P., Pagani, C., Pagano, I., Pailler, F., Palacin, H., Palaversa, L., Panahi, A., Pawlak, M., Piersimoni, A.M., Pineau, F.X., Plachy, E., Plum, G., Poggio, E., Poujoulet, E., Prša, A., Pulone, L., Racero, E., Ragaini, S., Rambaux, N., Ramos-Lerate, M., Regibo, S., Reylé, C., Riclet, F., Ripepi, V., Riva, A., Rivard, A., Rixon, G., Roegiers, T., Roelens, M., Romero-Gómez, M., Rowell, N., Royer, F., Ruiz-Dern, L., Sadowski, G., Sagristà Sellés, T., Sahlmann, J., Salgado, J., Salguero, E., Sanna, N., Santana- Ros, T., Sarasso, M., Savietto, H., Schultheis, M., Sciacca, E., Segol, M., Segovia, J.C., Ségransan, D., Shih, I.C., Siltala, L., Silva, A.F., Smart, R.L., Smith, K.W., Solano, E., Solitro, F., Sordo, R., Soria Nieto, S., Souchay, J., Spagna, A., Spoto, F., Stampa, U., Steele, I.A., Steidelmüller, H., Stephenson, C.A., Stoev, H., Suess, F.F., Surdej, J., Szabados, L., Szegedi-Elek, E., Tapiador, D., Taris, 2018. A\&A 616, A1. https://doi.org/10.1051/0004-6361/201833051.

Burton, W.B., 1988. The structure of our Galaxy derived from observations of neutral hydrogen. pp. 295-358.
Cantat-Gaudin, T., Krone-Martins, A., Sedaghat, N., Farahi, A., de Souza, R. S., Skalidis, R., Malz, A. I., Macêdo, S., Moews, B., Jordi, C., Moitinho, A., Castro-Ginard, A., Ishida, E. E. O., Heneka, C., Boucaud, A., Trindade, A. M. M., 2018. Gaia DR2 unravels incompleteness of nearby cluster population: New open clusters in the direction of Perseus. arXiv:1810.05494.

Cardelli, J.A., Clayton, G.C., Mathis, J.S., 1989. ApJ 345, 245-256. https://doi.org/10. 1086/167900.

Caswell, J.L., Haynes, R.F., 1987. A\&A 171, 261-276.

Chené, A.-N., Borissova, J., Bonatto, C., Majaess, D.J., Baume, G., Clarke, J.R.A., Kurtev, R., Schnurr, O., Bouret, J.-C., Catelan, M., Emerson, J.P., Feinstein, C., Geisler, D., de Grijs, R., Hervé, A., Ivanov, V.D., Kumar, M.S.N., Lucas, P., Mahy, L., Martins, F., Mauro, F., Minniti, D., Moni Bidin, C., 2013. A\&A 549, A98. https://doi.org/10. 1051/0004-6361/201220107.

Chené, A.-N., Borissova, J., Clarke, J.R.A., Bonatto, C., Majaess, D.J., Moni Bidin, C., Sale, S.E., Mauro, F., Kurtev, R., Baume, G., Feinstein, C., Ivanov, V.D., Geisler, D., Catelan, M., Minniti, D., Lucas, P., de Grijs, R., Kumar, M.S.N., 2012. A\&A 545, A54. https:// doi.org/10.1051/0004-6361/201219064.

Clemens, D.P., 1985. ApJ 295, 422-428. https://doi.org/10.1086/163386.

Corti, M.A., Baume, G.L., Panei, J.A., Suad, L.A., Testori, J.C., Borissova, J., Kurtev, R., Chené, A.N., Ramirez Alegría, S., 2016. A\&A 588, A63. https://doi.org/10.1051/ 0004-6361/201526121.

Cutri, R.M., et al., 2013. VizieR Online Data Catalog 2328.

Davies, B., de La Fuente, D., Najarro, F., Hinton, J.A., Trombley, C., Figer, D.F., Puga, E., 2012. MNRAS 419 (3), 1860-1870. https://doi.org/10.1111/j.1365-2966.2011. 19840.x.

Dickey, J.M., McClure-Griffiths, N.M., Gaensler, B.M., Green, A.J., 2003. ApJ 585, 801-822. https://doi.org/10.1086/346081.

Drew, J.E., Gonzalez-Solares, E., Greimel, R., Irwin, M.J., Küpcü Yoldas, A., Lewis, J., Barentsen, G., Eislöffel, J., Farnhill, H.J., 2014. MNRAS 440, 2036-3058. https://doi org/10.1093/mnras/stu394.

Dutra, C.M., Bica, E., Soares, J., Barbuy, B., 2003. A\&A 400, 533-539. https://doi.org/10. 1051/0004-6361:20030005.

Fich, M., Blitz, L., Stark, A.A., 1989. ApJ 342, 272-284. https://doi.org/10.1086/167591.

Figueira, M., López-Calderón, C., Bronfman, L., Zavagno, A., Hervías-Caimapo, C. Duronea, N., Nyman, L.-Å., 2019. A\&A 623, A141. https://doi.org/10.1051/0004$6361 / 201833001$.

Fish, V.L., Reid, M.J., Wilner, D.J., Churchwell, E., 2003. ApJ 587 (2), 701-713. https:// doi.org/10.1086/368284.

Gaia Collaboration, Prusti, T., de Bruijne, J.H.J., Brown, A.G.A., Vallenari, A., Babusiaux, C., Bailer-Jones, C.A.L., Bastian, U., Biermann, M., Evans, D.W., et al., 2016. A\&A 595, A1. https://doi.org/10.1051/0004-6361/201629272.

Hanson, M.M., Conti, P.S., Rieke, M.J., 1996. ApJS 107, 281. https://doi.org/10.1086/ 192366.

Hanson, M.M., Kudritzki, R.P., Kenworthy, M.A., Puls, J., Tokunaga, A.T., 2005. ApJS 161 (1), 154-170. https://doi.org/10.1086/444363.

Hook, I.M., Jørgensen, I., Allington-Smith, J.R., Davies, R.L., Metcalfe, N., Murowinski, R.G., Crampton, D., 2004. PASP 116, 425-440. https://doi.org/10.1086/383624.

Huang, M., Bania, T.M., Bolatto, A., Chamberlin, R.A., Ingalls, J.G., Jackson, J.M., Lane, A.P., Stark, A.A., Wilson, R.W., Wright, G.A., 1999. ApJ 517, 282-291. https://doi. org/10.1086/307194.

Jones, C., Dickey, J.M., 2012. ApJ 753, 62. https://doi.org/10.1088/0004-637X/753/ $1 / 62$.

Koenig, X.P., Leisawitz, D.T., Benford, D.J., Rebull, L.M., Padgett, D.L., Assef, R.J., 2012. ApJ 744, 130. https://doi.org/10.1088/0004-637X/744/2/130.

Kothes, R., Kerton, C.R., 2002. A\&A 390, 337-350. https://doi.org/10.1051/00046361:20020671.

Kurtz, S., Churchwell, E., Wood, D.O.S., 1994. ApJS 91, 659-712. https://doi.org/10. 1086/191952.

Lebrón, M.E., Rodríguez, L.F., Lizano, S., 2001. ApJ 560, 806-820. https://doi.org/10. $1086 / 322500$.

Liu, T., Kim, K.-T., Yoo, H., Liu, S.-y., Tatematsu, K., Qin, S.-L., Zhang, Q., Wu, Y., Wang, K., Goldsmith, P.F., Juvela, M., Lee, J.-E., Tóth, L.V., Mardones, D., Garay, G., Bronfman, L., Cunningham, M.R., Li, D., Lo, N., Ristorcelli, I., Schnee, S., 2016. ApJ 829, 59. https://doi.org/10.3847/0004-637X/829/2/59.

López, C., Bronfman, L., Nyman, L.-Å., May, J., Garay, G., 2011. A\&A 534, A131. https:// doi.org/10.1051/0004-6361/201015889.

McClure-Griffiths, N.M., Dickey, J.M., Gaensler, B.M., Green, A.J., Haverkorn, M., Strasser, S., 2005. ApJS 158, 178-187. https://doi.org/10.1086/430114.

Mezger, P.G., Henderson, A.P., 1967. ApJ 147, 471. https://doi.org/10.1086/149030.

Minniti, D., Lucas, P.W., Emerson, J.P., Saito, R.K., Hempel, M., Pietrukowicz, P., Ahumada, A.V., Alonso, M.V., Alonso-Garcia, J., Arias, J.I., Bandyopadhyay, R.M., Barbá, R.H., Barbuy, B., Bedin, L.R., Bica, E., Borissova, J., Bronfman, L., Carraro, G., Catelan, M., Clariá, J.J., Cross, N., de Grijs, R., Dékány, I., Drew, J.E., Fariña, C., Feinstein, C., Fernández Lajús, E., Gamen, R.C., Geisler, D., Gieren, W., Goldman, B., Gonzalez, O.A., Gunthardt, G., Gurovich, S., Hambly, N.C., Irwin, M.J., Ivanov, V.D., Jordán, A., Kerins, E., Kinemuchi, K., Kurtev, R., López-Corredoira, M., Maccarone, T., Masetti, N., Merlo, D., Messineo, M., Mirabel, I.F., Monaco, L., Morelli, L., Padilla, N., Palma, T., Parisi, M.C., Pignata, G., Rejkuba, M., Roman-Lopes, A., Sale, S.E., Schreiber, M.R., Schröder, A.C., Smith, M., Jr., L.S., Soto, M., Tamura, M., Tappert, C., Thompson, M.A., Toledo, I., Zoccali, M., Pietrzynski, G., 2010. New Astron. 15, 433-443. https://doi.org/10.1016/j.newast.2009.12.002.

Molina Lera, J.A., Baume, G., Gamen, R., 2018. MNRAS 480, 2386-2404. https://doi.org/ 10.1093/mnras/sty1874.

Moorwood, A., Cuby, J.-G., Lidman, C., 1998. The Messenger 91, 9-13.

Morales, E.F.E., Wyrowski, F., Schuller, F., Menten, K.M., 2013. A\&A 560, A76. https:// doi.org/10.1051/0004-6361/201321626. 
Quireza, C., Rood, R.T., Balser, D.S., Bania, T.M., 2006. ApJS 165, 338-359. https://doi. org/10.1086/503901.

Quireza, C., Rood, R.T., Bania, T.M., Balser, D.S., Maciel, W.J., 2006. ApJ 653, 1226-1240. https://doi.org/10.1086/508803.

Roman-Lopes, A., 2007. A\&A 471, 813-824. https://doi.org/10.1051/00046361:20066361.

Russeil, D., Zavagno, A., Mège, P., Poulin, Y., Molinari, S., Cambresy, L., 2017. A\&A 601, L5. https://doi.org/10.1051/0004-6361/201730540.

Sadler, E.M., Hunstead, R.W., 2001. SUMSS: Wide-field Radio Imaging of the Southern Sky. In: Clowes, R., Adamson, A., Bromage, G. (Eds.), The New Era of Wide Field Astronomy. Astronomical Society of the Pacific Conference Series 232. pp. 53.

Saito, R.K., Hempel, M., Minniti, D., Lucas, P.W., Rejkuba, M., Toledo, I., Gonzalez, O.A., Alonso-García, J., Irwin, M.J., Gonzalez-Solares, E., Hodgkin, S.T., 2012. A\&A 537, A107. https://doi.org/10.1051/0004-6361/201118407.

Skrutskie, M.F., Cutri, R.M., Stiening, R., Weinberg, M.D., Schneider, S., Carpenter, J.M., Beichman, C., Capps, R., Chester, T., Elias, J., 2006. AJ 131, 1163-1183. https://doi. org/10.1086/498708.

Spitzer, L., 1978. https://doi.org/10.1002/9783527617722.
Sternberg, A., Hoffmann, T.L., Pauldrach, A.W.A., 2003. ApJ 599, 1333-1343. https:// doi.org/10.1086/379506.

Stetson, P.B., 1987. PASP 99, 191-222. https://doi.org/10.1086/131977.

Stetson, P.B., 1992. More Experiments with DAOPHOT II and WF/PC Images. In: Worrall, D.M., Biemesderfer, C., Barnes, J. (Eds.), Astronomical Data Analysis Software and Systems I. Astronomical Society of the Pacific Conference Series 25. pp. 297.

Vallée, J.P., 2008. AJ 135, 1301-1310. https://doi.org/10.1088/0004-6256/135/4/ 1301.

Verma, R.P., Ghosh, S.K., Kulkarni, V.K., Ojha, D.K., 2003. Bull. Astron. Soc. India 31, 399-401.

Volk, K., Kwok, S., Stencel, R.E., Brugel, E., 1991. ApJS 77, 607-645. https://doi.org/10. 1086/191617.

Wienen, M., Wyrowski, F., Menten, K.M., Urquhart, J.S., Csengeri, T., Walmsley, C.M., Bontemps, S., Russeil, D., Bronfman, L., Koribalski, B.S., Schuller, F., 2015. A\&A 579, A91. https://doi.org/10.1051/0004-6361/201424802.

Wilson, T.L., Mezger, P.G., Gardner, F.F., Milne, D.K., 1970. A\&A 6, 364-384.

Wilson, T.L., Rohlfs, K., Hüttemeister, S., 2013. Tools of Radio Astronomy. https://doi. org/10.1007/978-3-642-39950-3. 